\title{
The Kirillov picture for the Wigner particle
}

\author{
J. M. Gracia-Bondía ${ }^{1}$, F. Lizzi ${ }^{2,3,4}$, J. C. Várilly ${ }^{5}$ and P. Vitale ${ }^{2,3}$ \\ ${ }^{1}$ Departamento de Física Teórica, Universidad de Zaragoza, Zaragoza 500o9, Spain \\ ${ }^{2}$ Dipartimento di Fisica “E. Pancini”, Università di Napoli Federico II, Italy \\ ${ }^{3}$ INFN Sezione di Napoli, Italy \\ ${ }^{4}$ Departament de Física Quàntica i Astrofísica \& Institut de Ciències del Cosmos, Universitat de Barcelona, Spain \\ ${ }^{5}$ Escuela de Matemática, Universidad de Costa Rica, San José 11501, Costa Rica
}

May 3, 2018

\begin{abstract}
We discuss the Kirillov method for massless Wigner particles, usually (mis)named "continuous spin" or "infinite spin" particles. These appear in Wigner's classification of the unitary representations of the Poincaré group, labelled by elements of the enveloping algebra of the Poincaré Lie algebra. Now, the coadjoint orbit procedure introduced by Kirillov is a prelude to quantization. Here we exhibit for those particles the classical Casimir functions on phase space, in parallel to quantum representation theory. A good set of position coordinates are identified on the coadjoint orbits of the Wigner particles; the stabilizer subgroups and the symplectic structures of these orbits are also described.
\end{abstract}

\section{Introduction}

The Wigner unitary representations (unirreps) of the Poincaré group [1], describing relativistic elementary particles, count, in our opinion, among the most important mathematical objects in the whole of physics. The Kirillov coadjoint orbit picture [2], on the other hand, has been known (for over a half century now) to link symplectic geometry with harmonic analysis. It is therefore surprising that relatively little work has been done so far to relate the Wigner unirreps with the phase space orbits (homogeneous symplectic manifolds endowed with a canonical Liouville measure) for the Poincaré group. The surprise only grows when one realizes that their correspondence is one-toone, particularly for maximal dimension orbits, like the ones considered here. A partial exception was the paper by J. F. Cariñena and two of us [3], devoted to phase spaces corresponding to massive particles. There, moreover, physical quantum averages were computed by means of phase space integrals, in a Wigner-Moyal approach.

It is our view that classical elementary relativistic phase spaces are objects as intrinsic as and perhaps more readily understandable than - the corresponding Wigner unirreps. Observables 
defined on elementary classical systems are useful proxies for operator quantities, since relativistic kinematics is the same for classical and quantum objects. In connection with the quantum field theory procedures, it should be noted that quantization of the coadjoint orbit picture runs parallel to induced representation theory [4], and allows to recover many of its results.

In this vein, we examine in this paper the phase space counterparts to Wigner's so-called "continuous spin" or "infinite spin" representations. These are misnomers (there is no such thing as continuous or infinite spin, although "unbounded" passes muster), so we shall call them simply Wigner particles, or WP for short. Till recently the latter had received scant attention, being curtly dismissed in textbooks. However, the path-breaking series of papers on the WP properties by Schuster and Toro [5-8] has awakened a lot of interest [9-11]; we retain chiefly [12], which introduces a quantum stress-energy-momentum tensor for the WP.

To the best of our knowledge, this article is the first study of the coadjoint orbits for the Wigner particle. We work out in detail the Poisson bracket structure for these lightlike systems. A crucial task is to find, and to establish the properties of, good position functions on the orbits.

The paper is organized as follows. Section 2 recalls the basics of the Kirillov procedure, as applied to the Poincaré group $\mathcal{P}$. We find the classical Casimir functions on phase space, in striking parallelism with quantum representation theory. Their values index the orbits corresponding to such representations, on which a convenient set of coordinates for the description of WPs is found. Subsection 2.3 dwells on an important kinematical question concerning massless particles.

In Section 3 the shape of the coadjoint orbits and their stabilizer subgroups are investigated. A surprisingly simple kinematics is thereby uncovered. We deal as well with the symplectic structure of those orbits and investigate the covariance properties of the coordinates under free motion. Section 4 is the Conclusion.

Readers are advised to check our conventions for relativistic kinematics in Appendix A, before tackling what follows.

\section{The Kirillov program for the Poincaré group}

The adjoint action $\underline{\text { Ad }}$ of $\mathcal{P}$ on its Lie algebra $\mathfrak{p}$ we compute as follows. The notation $\operatorname{ad}(X) Y:=[X, Y]$ for $X, Y \in \mathfrak{p}$ leads to $\operatorname{Ad}(\exp X) Y=e^{\operatorname{ad}(X)} Y=Y+[X, Y]+\frac{1}{2 !}[X,[X, Y]]+\cdots$. From this one can find $\operatorname{Ad}(\exp X) Y$ whenever $X=-a^{0} H, \boldsymbol{a} \cdot \boldsymbol{P}, \alpha \boldsymbol{m} \cdot \boldsymbol{L}$ or $\zeta \boldsymbol{n} \cdot \boldsymbol{K}$, with $\boldsymbol{m}$ and $\boldsymbol{n}$ denoting unit 3-vectors; here $Y=H \equiv P^{0}, P^{a}, L^{a}$ or $K^{a}$ are the respective generators of time and space translations, rotations and boosts.

For instance, if $X=\zeta \boldsymbol{n} \cdot \boldsymbol{K}, Y=H$, then

$$
\begin{aligned}
\operatorname{Ad}(\exp (\zeta \boldsymbol{n} \cdot \boldsymbol{K})) H & =H+\zeta[\boldsymbol{n} \cdot \boldsymbol{K}, H]+\frac{\zeta^{2}}{2 !}[\boldsymbol{n} \cdot \boldsymbol{K},[\boldsymbol{n} \cdot \boldsymbol{K}, H]]+\frac{\zeta^{3}}{3 !}[\boldsymbol{n} \cdot \boldsymbol{K},[\boldsymbol{n} \cdot \boldsymbol{K},[\boldsymbol{n} \cdot \boldsymbol{K}, H]]]+\cdots \\
& =H-\zeta \boldsymbol{n} \cdot \boldsymbol{P}+\frac{\zeta^{2}}{2 !} H-\frac{\zeta^{3}}{3 !} \boldsymbol{n} \cdot \boldsymbol{P}+\cdots=H \cosh \zeta-\boldsymbol{n} \cdot \boldsymbol{P} \sinh \zeta .
\end{aligned}
$$

In this way one obtains Table 1, exhibiting the adjoint action of $\mathcal{P}$ in a perspicuous manner. Explicitly for the rotation of angle $\alpha$ around the axis determined by the unit vector $\boldsymbol{m}$, acting on a vector $\boldsymbol{v}$ :

$$
R_{\alpha, \boldsymbol{m}} \boldsymbol{v}=\boldsymbol{v} \cos \alpha+\boldsymbol{m} \times \boldsymbol{v} \sin \alpha+(\boldsymbol{m} \cdot \boldsymbol{v}) \boldsymbol{m}(1-\cos \alpha) .
$$


Table 1: The adjoint action $\operatorname{Ad}(\exp X) Y$

\begin{tabular}{c|llll}
\hline \hline$Y \backslash X$ & $-a^{0} H$ & $\boldsymbol{a} \cdot \boldsymbol{P}$ & $\alpha \boldsymbol{m} \cdot \boldsymbol{L}$ & $\zeta \boldsymbol{n} \cdot \boldsymbol{K}$ \\
\hline$H$ & $H$ & $H$ & $H$ & $H \cosh \zeta-\boldsymbol{n} \cdot \boldsymbol{P} \sinh \zeta$ \\
$\boldsymbol{P}$ & $\boldsymbol{P}$ & $\boldsymbol{P}$ & $R_{\alpha, \boldsymbol{m}}^{-1} \boldsymbol{P}$ & $\boldsymbol{P}-H \boldsymbol{n} \sinh \zeta+(\boldsymbol{n} \cdot \boldsymbol{P}) \boldsymbol{n}(\cosh \zeta-1)$ \\
$\boldsymbol{L}$ & $\boldsymbol{L}$ & $\boldsymbol{L}-\boldsymbol{a} \times \boldsymbol{P}$ & $R_{\alpha, \boldsymbol{m}}^{-1} \boldsymbol{L}$ & $\boldsymbol{L} \cosh \zeta-\boldsymbol{n} \times \boldsymbol{K} \sinh \zeta-(\boldsymbol{n} \cdot \boldsymbol{L}) \boldsymbol{n}(\cosh \zeta-1)$ \\
$\boldsymbol{K}$ & $\boldsymbol{K}-a^{0} \boldsymbol{P}$ & $\boldsymbol{K}+H \boldsymbol{a}$ & $R_{\alpha, \boldsymbol{m}}^{-1} \boldsymbol{K}$ & $\boldsymbol{K} \cosh \zeta+\boldsymbol{n} \times \boldsymbol{L} \sinh \zeta-(\boldsymbol{n} \cdot \boldsymbol{K}) \boldsymbol{n}(\cosh \zeta-1)$ \\
\hline \hline
\end{tabular}

Table 2: The coadjoint action Coad $(\exp X) y$

\begin{tabular}{c|llll}
\hline \hline$y \backslash X$ & $-a^{0} H$ & $\boldsymbol{a} \cdot \boldsymbol{P}$ & $\alpha \boldsymbol{m} \cdot \boldsymbol{L}$ & $\zeta \boldsymbol{n} \cdot \boldsymbol{K}$ \\
\hline$h$ & $h$ & $h$ & $h$ & $h \cosh \zeta+\boldsymbol{n} \cdot \boldsymbol{p} \sinh \zeta$ \\
$\boldsymbol{p}$ & $\boldsymbol{p}$ & $\boldsymbol{p}$ & $R_{\alpha, \boldsymbol{m}} \boldsymbol{p}$ & $\boldsymbol{p}+h \boldsymbol{n} \sinh \zeta+(\boldsymbol{n} \cdot \boldsymbol{p}) \boldsymbol{n}(\cosh \zeta-1)$ \\
$\boldsymbol{l}$ & $\boldsymbol{l}$ & $\boldsymbol{l}+\boldsymbol{a} \times \boldsymbol{p}$ & $R_{\alpha, \boldsymbol{m}} \boldsymbol{l}$ & $\boldsymbol{l} \cosh \zeta+\boldsymbol{n} \times \boldsymbol{k} \sinh \zeta-(\boldsymbol{n} \cdot \boldsymbol{l}) \boldsymbol{n}(\cosh \zeta-1)$ \\
$\boldsymbol{k}$ & $\boldsymbol{k}+a^{0} \boldsymbol{p}$ & $\boldsymbol{k}-h \boldsymbol{a}$ & $R_{\alpha, \boldsymbol{m}} \boldsymbol{k}$ & $\boldsymbol{k} \cosh \zeta-\boldsymbol{n} \times \boldsymbol{l} \sinh \zeta-(\boldsymbol{n} \cdot \boldsymbol{k}) \boldsymbol{n}(\cosh \zeta-1)$ \\
\hline \hline
\end{tabular}

The coadjoint action $\underline{\text { Coad }}$ of $\mathcal{P}$ on elements of the Lie coalgebra $\mathfrak{p}^{*}$,

$$
\langle\operatorname{Coad}(\exp X) u, Y\rangle:=\langle u, \operatorname{Ad}(\exp (-X)) Y\rangle \quad \text { for } \quad u \in \mathfrak{p}^{*},
$$

can now be derived immediately. Let $h$ be the linear coordinate on $\mathfrak{p}^{*}$ associated to $H$, and similarly let $p^{a}, l^{a}, k^{a}$ be the coordinates associated to $P^{a}, L^{a}, K^{a}(a=1,2,3)$. The action is given in these coordinates by Table 2 .

We shall need the natural Lie-Poisson bracket on $\mathfrak{p}^{*}$ : given $f \in C^{\infty}\left(\mathfrak{p}^{*}\right)$, one can regard $d f(u)$ as an element of the Lie algebra, and one obtains:

$$
\{f, g\}(u):=\langle u,[d f(u), d g(u)]\rangle=c^{\alpha \beta} \gamma \frac{\partial f(u)}{\partial u^{\alpha}} \frac{\partial g(u)}{\partial u^{\beta}} u^{\gamma},
$$

where the $c^{\alpha \beta}{ }_{\gamma}$ are the structure constants of $\mathfrak{p}$. Therefore, taking $(h, \boldsymbol{p}, \boldsymbol{l}, \boldsymbol{k})$ as cartesian coordinates on $\mathfrak{p}^{*}$, their Poisson brackets are given directly by the commutation relations (A.1) among the corresponding Lie algebra generators. For reference: ${ }^{1}$

$$
\begin{aligned}
& \left\{l^{a}, l^{b}\right\}=\varepsilon^{a b}{ }_{c} l^{c}, \quad\left\{l^{a}, k^{b}\right\}=\varepsilon^{a b}{ }_{c} k^{c}, \quad\left\{k^{a}, k^{b}\right\}=-\varepsilon^{a b}{ }_{c} l^{c}, \\
& \left\{l^{a}, p^{b}\right\}=\varepsilon^{a b}{ }_{c} p^{c}, \quad\left\{p^{b}, k^{a}\right\}=\delta^{a b} h, \quad\left\{h, k^{a}\right\}=p^{a} .
\end{aligned}
$$

\subsection{The Casimir functions}

The Lie-Poisson bracket (2.2) restricts to symplectic structures on the coadjoint orbits foliating it. Generally speaking, the orbits arise as level sets of two "Casimir functions" $C_{1}, C_{2}$ on p* that

${ }^{1}$ For the problem at hand, the nonrelativistic notation, which separates rotations from boosts and time from space translations, is more transparent. 
are invariant by the coadjoint action. These are easy to obtain explicitly. Let $p=(h, \boldsymbol{p})$ be the energy-momentum 4-vector and $w=\left(w^{0}, \boldsymbol{w}\right)$ the phase-space "Pauli-Lubański" 4-vector, defined by

$$
w^{0}=\boldsymbol{l} \cdot \boldsymbol{p} ; \quad \boldsymbol{w}=\boldsymbol{k} \times \boldsymbol{p}+h \boldsymbol{l} .
$$

Clearly $p$ and $w$ are orthogonal in the Minkowski sense: $(p w)=0$. From Table 2 , with a little work, one verifies that $\left(w^{0}, \boldsymbol{w}\right)$ transforms like $(h, \boldsymbol{p})$ under the coadjoint action. In particular, under the boost $\operatorname{Coad}(\exp (\zeta \boldsymbol{n} \cdot \boldsymbol{K}))$ :

$$
\begin{aligned}
w^{0} & \mapsto w^{0} \cosh \zeta+\boldsymbol{n} \cdot \boldsymbol{w} \sinh \zeta, \\
\boldsymbol{w} & \mapsto \boldsymbol{w}+w^{0} \boldsymbol{n} \sinh \zeta+(\boldsymbol{n} \cdot \boldsymbol{w}) \boldsymbol{n}(\cosh \zeta-1) .
\end{aligned}
$$

Lemma 1. The Poisson brackets of the components of $w$ with the basic variables are given by:

$$
\begin{aligned}
& \left\{h, w^{\mu}\right\}=0, \quad\left\{k^{a}, w^{0}\right\}=-w^{a}, \quad\left\{l^{a}, w^{0}\right\}=0, \\
& \left\{p^{a}, w^{\mu}\right\}=0, \quad\left\{k^{a}, w^{b}\right\}=-\delta^{a b} w^{0}, \quad\left\{l^{a}, w^{b}\right\}=\varepsilon^{a b}{ }_{c} w^{c} \text {; }
\end{aligned}
$$

and among the components, the brackets are:

$$
\left\{w^{0}, w^{a}\right\}=(\boldsymbol{w} \times \boldsymbol{p})^{a}, \quad\left\{w^{a}, w^{b}\right\}=\varepsilon^{a b}{ }_{c}\left(h w^{c}-w^{0} p^{c}\right) .
$$

Proof. By direct computation, using (2.3).

Proposition 2. The Casimir functions we seek are

$$
C_{1}:=(p p)=h^{2}-|\boldsymbol{p}|^{2}, \quad C_{2}:=(w w)=(\boldsymbol{l} \cdot \boldsymbol{p})^{2}-|\boldsymbol{k} \times \boldsymbol{p}+h \boldsymbol{l}|^{2} .
$$

Proof. The relations (2.4) follow from (2.3). That $\left\{h, w^{0}\right\}=0$ is clear; the others are:

$$
\begin{aligned}
\left\{h, w^{a}\right\} & =\left\{h, \varepsilon_{b c}^{a} k^{b} p^{c}\right\}=\varepsilon_{b c}^{a} p^{b} p^{c}=0, \\
\left\{p^{a}, w^{0}\right\} & =\delta_{b d}\left\{p^{a}, l^{b}\right\} p^{d}=\delta_{b d} \varepsilon_{b c}^{a} p^{c} p^{d}=0, \\
\left\{p^{a}, w^{b}\right\} & =\left\{p^{a}, \varepsilon^{b}{ }_{c d} k^{c} p^{d}+h l^{b}\right\}=\varepsilon^{b}{ }_{a d} h p^{d}+\varepsilon^{a b}{ }_{c} h p^{c}=0, \\
\left\{k^{a}, w^{0}\right\} & =\left\{k^{a}, \delta_{b d} l^{b} p^{d}\right\}=\delta_{b d}\left(\left\{k^{a}, l^{b}\right\} p^{d}+l^{b}\left\{k^{a}, p^{d}\right\}\right)=\varepsilon^{a}{ }_{b c} k^{c} p^{b}-h l^{a}=-w^{a}, \\
\left\{k^{a}, w^{a}\right\} & =\left\{k^{a}, \varepsilon^{a} b d k^{b} p^{d}+h l^{a}\right\}=-\varepsilon^{a} b d \varepsilon^{a b}{ }_{c} l^{c} p^{d}-l^{a} p^{a}=-\delta_{c d} l^{c} p^{d}=-w^{0}, \\
\left\{k^{a}, w^{b}\right\} & =\left\{k^{a}, \varepsilon^{b}{ }_{c d} k^{c} p^{d}+h l^{b}\right\}=-\varepsilon^{b}{ }_{c d} \varepsilon^{a c}{ }_{e} l^{e} p^{d}-\varepsilon^{b}{ }_{c a} k^{c} h-l^{b} p^{a}+\varepsilon^{a b}{ }_{c} k^{c} h \\
& =l^{b} p^{a}-l^{b} p^{a}=0 \quad \text { if } a \neq b, \\
\left\{l^{a}, w^{0}\right\} & =\delta_{b d}\left\{l^{a}, l^{b} p^{d}\right\}=\varepsilon^{a}{ }_{b c}\left(l^{c} p^{b}+l^{b} p^{c}\right)=0, \\
\left\{l^{a}, w^{b}\right\} & =\left\{l^{a},(\boldsymbol{k} \times \boldsymbol{p})^{b}\right\}+h\left\{l^{a}, l^{b}\right\}=\varepsilon^{a b}{ }_{c}\left((\boldsymbol{k} \times \boldsymbol{p})^{c}+h l^{c}\right)=\varepsilon^{a b}{ }_{c} w^{c} ;
\end{aligned}
$$

From these brackets, (2.5) follows easily:

$$
\begin{aligned}
& \left\{w^{0}, w^{a}\right\}=\left\{w^{0}, \varepsilon^{a}{ }_{b d} k^{b} p^{d}+h l^{a}\right\}=\varepsilon^{a}{ }_{b d} w^{b} p^{d}=(\boldsymbol{w} \times \boldsymbol{p})^{a}, \\
& \left\{w^{a}, w^{b}\right\}=\left\{w^{a}, \varepsilon^{b}{ }_{c d} k^{c} p^{d}+h l^{b}\right\}=\varepsilon^{b}{ }_{c d} \delta^{a c} w^{0} p^{d}+\varepsilon^{a b}{ }_{c} h w^{c}=\varepsilon^{a b}{ }_{c}\left(h w^{c}-w^{0} p^{c}\right) .
\end{aligned}
$$

That $C_{1}$ is a Casimir hardly needs proof. From formulas (2.4) it follows that

$$
\left\{k^{a}, C_{2}\right\}=0=\left\{l^{a}, C_{2}\right\}
$$

and Proposition 2 is proved. 


\subsection{Searching for position coordinates}

In order to find and study the orbits corresponding to the WP, it is natural to look for global position functions.

Let us quickly review the massive case, to better understand the situation. That is, we restrict ourselves to orbits for which $C_{1}>0$, writing $C_{1}=m^{2}$ and we decide on $h=+\sqrt{m^{2}+|\boldsymbol{p}|^{2}}$. Note that $C_{1} \geq 0$ implies $C_{2} \leq 0$. Let $\kappa:=(m, \mathbf{0})$ be the vertex of the forward hyperboloid given by $p^{2}=m^{2}$. Consider the standard Lorentz boost $L_{p}$ which takes $(1, \mathbf{0})$ to $u:=p / m$; its relation to $\operatorname{Coad}(\exp (\zeta \boldsymbol{n} \cdot \boldsymbol{K}))$ is given by

$$
\boldsymbol{n}:=\frac{\boldsymbol{u}}{|\boldsymbol{u}|}=\frac{\boldsymbol{u}}{\sqrt{\left(u^{0}\right)^{2}-1}} ; \quad u^{0}=: \cosh \zeta \quad(\zeta \geq 0) .
$$

One thus obtains

$$
L_{p} a=\left(\frac{h a^{0}+\boldsymbol{p} \cdot \boldsymbol{a}}{m}, \boldsymbol{a}+\left(\frac{a^{0}}{m}+\frac{\boldsymbol{p} \cdot \boldsymbol{a}}{m(m+h)}\right) \boldsymbol{p}\right) .
$$

Now $0=(p w)=\left(L_{p}^{-1} p L_{p}^{-1} w\right)=\left(\kappa L_{p}^{-1} w\right)$. This means that $L_{p}^{-1} w=(0, m s)$ for some 3-vector $\boldsymbol{s}$. The Casimir $C_{2}=(w w)=\left(L_{p}^{-1} w L_{p}^{-1} w\right)=-m^{2}|s|^{2} \leq 0$ is constant on any orbit; thus, if $C_{2}<0$ we interpret $s$ as the spin vector. From $(0, m s)=L_{p}^{-1} w$ one derives the relation:

$$
m \boldsymbol{s}=\boldsymbol{w}-\frac{w^{0}}{m+h} \boldsymbol{p}=\boldsymbol{w}-\frac{(\boldsymbol{w} \cdot \boldsymbol{p})}{h(m+h)} \boldsymbol{p} .
$$

For fixed $C_{1}$ and $C_{2}$, an orbit $\mathcal{O}_{m s+}$ has been obtained. One naturally takes as coordinates on it the momenta $\boldsymbol{p}$ and spherical coordinates arising from $m \boldsymbol{s}$. Three more come from a position triplet $\boldsymbol{q}$, given by [3]:

$$
\boldsymbol{q}:=-\frac{\boldsymbol{k}}{h}-\frac{\boldsymbol{p} \times \boldsymbol{w}}{m h(m+h)}=-\frac{\boldsymbol{k}}{h}-\frac{\boldsymbol{p} \times \boldsymbol{s}}{h(m+h)} .
$$

The expressions of the $\mathfrak{p}^{*}$-coordinates $\boldsymbol{l}, \boldsymbol{k}$ in terms of the $\mathcal{O}_{m s+}$ coordinates $(\boldsymbol{q}, \boldsymbol{p}, \boldsymbol{s})$ over the orbit are:

$$
\boldsymbol{k}=-h \boldsymbol{q}-\frac{\boldsymbol{p} \times \boldsymbol{s}}{m+h}, \quad \boldsymbol{l}=\boldsymbol{q} \times \boldsymbol{p}+\boldsymbol{s} .
$$

Clearly $\mathcal{O}_{m s+}$ is homeomorphic to $\mathbb{R}^{6} \times \mathbb{S}^{2}$, with isotropy (or stability) group isomorphic to $\mathbb{R} \times S O(2)$ - say, the subgroup fixing $(\mathbf{0}, \mathbf{0}, s)$ generated by time translations $\exp \left(-a^{0} H\right)$ and rotations $R_{\alpha, s}$, see [3, Eqn. (30)]. ${ }^{2}$ The degenerate "scalar" case $C_{2}=0$ gives 6-dimensional orbits $\mathcal{O}_{m 0+}$, homeomorphic to $\mathbb{R}^{6}$.

Using (2.2) together with the commutation relations, one verifies that $\left\{q^{a}, p^{a}\right\}$ are canonical coordinates, and that $\left\{s^{a}, s^{b}\right\}=\varepsilon^{a b}{ }_{c} s^{c} ;\left\{s^{a}, q^{b}\right\}=0=\left\{s^{a}, p^{b}\right\}$. These coordinates, however, are not particularly useful. They do not transform covariantly for $s \neq 0$; in [3] they were replaced by others that do so. They are certainly useless to study the massless limit. We shall come back to the question of different sets of position coordinates repeatedly.

We turn now to massless particles, the WP in particular. Over fifty years ago, Wightman wrote a remarkable paper [14] proving that quantum spinning massless systems like the photon are not

\footnotetext{
${ }^{2}$ Coadjoint orbits always have even dimension [2]; and their isotropy groups for maximal dimensional orbits are always abelian [13].
} 
localizable, in the sense that for them the action of the Euclidean group cannot be realized on a set of three position coordinates in the standard way. However, he assumes commutativity of those variables. In the first chapter of his book [15], Schwinger disclosed a view on relativistic position operators that can be understood as a retort to Wightman's. In fact, allowing for noncommutativity, there are position operators for photons and other fixed-helicity particles, as well as for the WP, with the correct transformation properties.

It is both instructive and convenient in our phase-space context to seek a position vector for the massive case with good limit properties as $m \downarrow 0$. Thus we adopt a set of coordinates $\boldsymbol{r}$ suggested by Schwinger's ideas, ${ }^{3}$ provided by

$$
\boldsymbol{r}:=-\frac{\boldsymbol{k}}{h}+\frac{\boldsymbol{p} \times \boldsymbol{w}}{h^{2}(m+h)} .
$$

Notice that as $m \downarrow 0$, the right hand side of Eqn. (2.6) becomes:

$$
\boldsymbol{t}:=\boldsymbol{w}-\frac{(\boldsymbol{w} \cdot \boldsymbol{p}) \boldsymbol{p}}{h^{2}}=\boldsymbol{w}-\frac{w^{0}}{h} \boldsymbol{p} .
$$

Now we consider the WP case, where by definition $C_{1}=m^{2}=0$ and $C_{2}=w^{2}=:-\rho^{2}<0$. Note that, since $(w p)=0$, this entails $p \nmid w$ and therefore $\boldsymbol{t} \neq \mathbf{0}$; also, $\boldsymbol{p} \neq \mathbf{0}$ since $C_{1}=0$ but $w^{2} \neq 0$. Moreover, $(w p)=0$ implies that $\boldsymbol{t} \perp \boldsymbol{p}$ and $|\boldsymbol{t}|^{2}=\rho^{2}$; and thus $|(\boldsymbol{p} / h) \times \boldsymbol{t}|^{2}=\rho^{2}$, as well. ${ }^{4}$

Position coordinates for the WP are given by

$$
\boldsymbol{r}:=-\frac{\boldsymbol{k}}{h}+\frac{\boldsymbol{p} \times \boldsymbol{w}}{h^{3}}=-\frac{\boldsymbol{k}}{h}+\frac{\boldsymbol{p} \times \boldsymbol{t}}{h^{3}}=\frac{(\boldsymbol{p} \cdot \boldsymbol{k}) \boldsymbol{p}}{h^{3}}+\frac{\boldsymbol{p} \times \boldsymbol{l}}{h^{2}} .
$$

Introduce the notation $\lambda$ for the important helicity variable:

$$
\lambda:=\frac{\boldsymbol{l} \cdot \boldsymbol{p}}{h}=\frac{w^{0}}{h} .
$$

One readily obtains the basic coalgebra functions $\boldsymbol{k}, \boldsymbol{l}$ in terms of the new $(\boldsymbol{r}, \boldsymbol{p}, \lambda, \boldsymbol{t})$ set of variables. ${ }^{5}$

$$
\boldsymbol{w}=\lambda \boldsymbol{p}+\boldsymbol{t}, \quad \text { thus } \quad \boldsymbol{k}=-h \boldsymbol{r}+\frac{\boldsymbol{p} \times \boldsymbol{t}}{h^{2}}, \quad \boldsymbol{l}=h^{-1}(\boldsymbol{w}-\boldsymbol{k} \times \boldsymbol{p})=\boldsymbol{r} \times \boldsymbol{p}+\frac{\lambda}{h} \boldsymbol{p} .
$$

We require to have available the Poisson brackets involving the new variables. Remark first that $\left\{\lambda, p^{a}\right\}=\left\{\lambda, l^{a}\right\}=0$, and that the first relation in (2.5) can be rewritten as:

$$
\left\{w^{0}, t^{a}\right\}=\left\{w^{0}, w^{a}\right\}=-(\boldsymbol{p} \times \boldsymbol{w})^{a}=-(\boldsymbol{p} \times \boldsymbol{t})^{a} .
$$

Lemma 3. The helicity and the 3-vector t have these Poisson brackets:

$$
\begin{aligned}
\left\{\lambda, k^{a}\right\} & =t^{a} / h, & \left\{k^{a}, t^{b}\right\} & =t^{a} p^{b} / h, \\
\{\lambda, \boldsymbol{t}\} & =-\boldsymbol{p} / h \times \boldsymbol{t}, & \left\{l^{a}, t^{b}\right\} & =\varepsilon^{a b}{ }_{c} t^{c}, \\
\{\lambda, \boldsymbol{p} / h \times \boldsymbol{t}\} & =\boldsymbol{t}, & \left\{p^{a}, t^{b}\right\} & =\left\{t^{a}, t^{b}\right\}=0 .
\end{aligned}
$$

\footnotetext{
${ }^{3} \mathrm{~A}$ discussion closer to the original treatment is given in Appendix B.

${ }^{4}$ The parameter $\rho$ has the physical dimension of energy. Orbits with different values of $\rho$ correspond to different particles.

${ }^{5} \mathrm{We}$ have seen that the last of these is constrained by $\boldsymbol{t} \cdot \boldsymbol{p}=0$ and $\boldsymbol{t}^{2}=\rho^{2}$, so certainly these maximal orbits are 8-dimensional.
} 
Proof. The first relation comes from

$$
\left\{\lambda, k^{a}\right\}=\left\{w^{0} h^{-1}, k^{a}\right\}=\left\{w^{0}, k^{a}\right\} h^{-1}-w^{0} h^{-1}\left\{h, k^{a}\right\} h^{-1}=\left(w^{a}-\lambda p^{a}\right) / h=t^{a} / h .
$$

The second one is given by

$$
\left\{k^{a}, t^{b}\right\}=\left\{k^{a}, w^{b}-w^{0} p^{b} h^{-1}\right\}=-\delta^{a b} w^{0}+w^{a} p^{b} h^{-1}+\delta^{a b} w^{0}-w^{0} p^{b} p^{a} h^{-2}=t^{a} p^{b} / h .
$$

Furthermore,

$$
\{\lambda, \boldsymbol{t}\}=\{\lambda, \boldsymbol{w}\}=\{\lambda, \boldsymbol{k} \times \boldsymbol{p}\}=\{\lambda, \boldsymbol{k}\} \times \boldsymbol{p}=-\boldsymbol{p} / h \times \boldsymbol{t},
$$

and thus also

$$
\{\lambda, \boldsymbol{p} / h \times \boldsymbol{t}\}=\boldsymbol{p} / h \times\{\lambda, \boldsymbol{t}\}=-\boldsymbol{p} \times(\boldsymbol{p} \times \boldsymbol{t}) / h^{2}=\left(|\boldsymbol{p}|^{2} / h^{2}\right) \boldsymbol{t}=\boldsymbol{t},
$$

on account of $(p p)=0$ and $\boldsymbol{p} \cdot \boldsymbol{t}=0$. From equations (2.3) and (2.4), we see at once that $\left\{l^{a}, t^{b}\right\}=\varepsilon^{a b}{ }_{c} t^{c}$. Similarly, $\left\{p^{a}, t^{b}\right\}=0$ follows from (2.4) and (2.9).

Note that the second relation of (2.5) can now be shortened to $\left\{w^{a}, w^{b}\right\}=\varepsilon^{a b}{ }_{c} h t^{c}$. In consequence, the components of $\boldsymbol{t}$ Poisson-commute:

$$
\begin{aligned}
\left\{t^{a}, t^{b}\right\} & =\left\{w^{a}, w^{b}\right\}-p^{a}\left\{\lambda, w^{b}\right\}-\left\{w^{a}, \lambda\right\} p^{b} \\
& =\varepsilon^{a b}{ }_{c} h t^{c}+p^{a}(\boldsymbol{p} / h \times \boldsymbol{t})^{b}-(\boldsymbol{p} / h \times \boldsymbol{t})^{a} p^{b} \\
& =\varepsilon^{a b}{ }_{c}\left(h t^{c}+(\boldsymbol{p} \times(\boldsymbol{p} \times \boldsymbol{t}))^{c} / h\right)=0 .
\end{aligned}
$$

Lemma 4. The Poisson brackets involving the position variables are given by:

$$
\begin{aligned}
& \left\{r^{a}, p^{b}\right\}=\delta^{a b}, \quad\left\{r^{a}, h\right\}=p^{a} / h, \quad\left\{\boldsymbol{r}, w^{0}\right\}=\boldsymbol{w}_{\|} / h, \quad\left\{r^{a}, \lambda\right\}=0, \\
& \left\{r^{a}, t^{b}\right\}=-t^{a} p^{b} / h^{2}, \quad\left\{l^{a}, r^{b}\right\}=\varepsilon^{a b}{ }_{c} r^{c}, \quad\left\{r^{a}, r^{b}\right\}=-\varepsilon^{a b}{ }_{c} \lambda p^{c} / h^{3},
\end{aligned}
$$

and also:

$$
\left\{r^{a}, w^{b}\right\}=\lambda \delta^{a b}-t^{a} p^{b} / h^{2}, \quad\left\{r^{a}, k^{b}\right\}=-p^{a} r^{b} / h+\varepsilon^{a b}{ }_{c}\left(\lambda p^{c}-t^{c}\right) / h^{2}-2 p^{a}(\boldsymbol{p} \times \boldsymbol{t})^{b} / h^{4} .
$$

Proof. A few of these follow directly from the basic Poisson brackets (2.3). Indeed, since $\left\{h, p^{b}\right\}=0$ and $\left\{(\boldsymbol{p} \times \boldsymbol{w})^{a}, p^{b}\right\}=0$, the first reduces to $\left\{r^{a}, p^{b}\right\}=-\left\{k^{a}, p^{b}\right\} / h=\delta^{a b}$. That is to say, $\boldsymbol{r}$ and $\boldsymbol{p}$ are conjugates, as is naturally demanded of a position vector.

In like manner, $\left\{r^{a}, h\right\}=-\left\{k^{a} / h, h\right\}=p^{a} / h$. Also from (2.3) we get:

$$
\left\{l^{a}, r^{b}\right\}=-\left\{l^{a}, k^{b}\right\} / h+\left\{l^{a},(\boldsymbol{p} \times \boldsymbol{w})^{b}\right\} / h^{3}=\varepsilon^{a b}{ }_{c}\left(-k^{c} / h+(\boldsymbol{p} \times \boldsymbol{w})^{c} / h^{3}\right)=\varepsilon^{a b}{ }_{c} r^{c} .
$$

Three more relations follow from (2.12):

$$
\begin{aligned}
\left\{\boldsymbol{r}, w^{0}\right\} & =-\left\{\boldsymbol{k}, w^{0}\right\} / h+\left\{\boldsymbol{p} \times \boldsymbol{t}, w^{0}\right\} / h^{3}=\boldsymbol{w} / h+\boldsymbol{p} \times(\boldsymbol{p} \times \boldsymbol{t}) / h^{3}=\boldsymbol{w} / h-\boldsymbol{t} / h=\boldsymbol{w}_{\|} / h, \\
\{\boldsymbol{r}, \lambda\} & =-\{\boldsymbol{k}, \lambda\} / h+\{\boldsymbol{p} / h \times \boldsymbol{t}, \lambda\} / h^{2}=-\boldsymbol{t} / h^{2}+\boldsymbol{t} / h^{2}=\mathbf{0}, \\
\left\{r^{a}, t^{b}\right\} & =-\left\{k^{a} / h, t^{b}\right\}=-t^{a} p^{b} / h^{2} .
\end{aligned}
$$

These in turn imply that

$$
\left\{r^{a}, w^{b}\right\}=\left\{r^{a}, \lambda p^{b}+t^{b}\right\}=\left\{r^{a}, \lambda\right\} p^{b}+\lambda\left\{r^{a}, p^{b}\right\}+\left\{r^{a}, t^{b}\right\}=\lambda \delta^{a b}-t^{a} p^{b} / h^{2} .
$$


The last relation in (2.13) becomes a sum of three terms:

where

$$
\left\{r^{a}, r^{b}\right\}=\left\{k^{a} / h, k^{b} / h\right\}-\left\{k^{a} / h,(\boldsymbol{p} \times \boldsymbol{t})^{b} / h^{3}\right\}-\left\{(\boldsymbol{p} \times \boldsymbol{t})^{a} / h^{3}, k^{b} / h\right\},
$$

$$
\begin{aligned}
\left\{k^{a} / h, k^{b} / h\right\} & =\left\{k^{a}, k^{b}\right\} h^{-2}+k^{a}\left\{h^{-1}, k^{b}\right\} h^{-1}+k^{b}\left\{k^{a}, h^{-1}\right\} h^{-1} \\
& =-\varepsilon^{a b}{ }_{c} l^{c} / h^{2}-k^{a} p^{b} / h^{3}+k^{b} p^{a} / h^{3}=-\varepsilon^{a b}{ }_{c} w^{c} / h^{3} ; \\
-\left\{k^{a} / h,(\boldsymbol{p} \times \boldsymbol{t})^{b} / h^{3}\right\} & =\varepsilon^{b}{ }_{d e}\left\{k^{a}, t^{d} p^{e} / h^{3}\right\} / h \\
& =\varepsilon^{b}{ }_{d e}\left(\left\{k^{a}, t^{d}\right\} p^{e} / h^{4}+t^{d}\left\{k^{a}, p^{e}\right\} / h^{4}-3 t^{d} p^{e}\left\{k^{a}, h\right\} / h^{5}\right) \\
& =\varepsilon^{b}{ }_{d e}\left(t^{a} p^{d} p^{e} / h^{4}-\delta^{a e} t^{d} / h^{3}+3 t^{d} p^{e} p^{a} / h^{5}\right) \\
& =-\varepsilon^{a b}{ }_{c} t^{c} / h^{3}-3(\boldsymbol{p} \times \boldsymbol{t})^{b} p^{a} / h^{5} ;
\end{aligned}
$$

and similarly, $-\left\{(\boldsymbol{p} \times \boldsymbol{t})^{a} / h^{3}, k^{b} / h\right\}=-\varepsilon^{a b}{ }_{c} t^{c} / h^{3}-3(\boldsymbol{p} \times \boldsymbol{t})^{a} p^{b} / h^{5}$. Therefore,

$$
\left\{r^{a}, r^{b}\right\}=-\varepsilon^{a b}{ }_{c}\left(h^{2} w^{c}+2 h^{2} t^{c}+3(\boldsymbol{p} \times(\boldsymbol{p} \times \boldsymbol{t}))^{c}\right) / h^{5}=\varepsilon^{a b}{ }_{c}\left(t^{c}-w^{c}\right) / h^{3}=-\varepsilon^{a b}{ }_{c} \lambda p^{c} / h^{3} .
$$

Using Eqs. (2.11) and (2.13), it now follows that

$$
\begin{aligned}
\left\{r^{a}, k^{b}\right\} & =-\left\{r^{a}, h r^{b}\right\}+\left\{r^{a}, \varepsilon_{d e}^{b} p^{d} t^{e} / h^{2}\right\} \\
& =-\left\{r^{a}, h\right\} r^{b}-h\left\{r^{a}, r^{b}\right\}+\varepsilon_{d e}^{b}\left(\left\{r^{a}, p^{d}\right\} t^{e} / h^{2}+p^{d}\left\{r^{a}, t^{e}\right\} / h^{2}-2 p^{d} t^{e}\left\{r^{a}, h\right\} / h^{3}\right) \\
& =-p^{a} r^{b} / h+\varepsilon^{a b}\left(\lambda p^{c}-t^{c}\right) / h^{2}-2 p^{a}(\boldsymbol{p} \times \boldsymbol{t})^{b} / h^{4} .
\end{aligned}
$$

The formulas of the previous lemmata remain valid for the fixed-helicity situations, by replacing $\boldsymbol{t}$ by $\mathbf{0}$ and "freezing" $\lambda$ to a given value. The commutation relations $\left\{r^{a}, r^{b}\right\}=-\varepsilon^{a b}{ }_{c} \lambda p^{c} / h^{3}$ were actually found for the latter situation already in [16].

\subsection{On the Wigner rotation}

Let us return briefly to the massive case, $m>0$. Let $w^{\prime}=\Lambda w$, and consider accordingly

$$
\left(0, m \mathbf{s}^{\prime}\right):=L_{\Lambda p}^{-1} w^{\prime}=L_{\Lambda p}^{-1} \Lambda L_{p}(0, m \boldsymbol{s}) .
$$

This transformation is just the Wigner rotation $g(\Lambda, p)$. The spin's axis of rotation is given by $\boldsymbol{p} \times \boldsymbol{n}$ for a boost $\Lambda$ in the direction of $\boldsymbol{n}$ : when the boost is parallel to the momentum $\boldsymbol{p}$, the Wigner rotation is trivial. With $\boldsymbol{m}:=(\boldsymbol{p} \times \boldsymbol{n}) /|\boldsymbol{p} \times \boldsymbol{n}|$, the formula is [3]:

$$
\boldsymbol{s}^{\prime}=g(\Lambda, p) \boldsymbol{s}=R_{\delta, \boldsymbol{m}} \boldsymbol{s}=\boldsymbol{s}+(\boldsymbol{m} \times \boldsymbol{s}) \sin \delta-(\boldsymbol{s}-(\boldsymbol{m} \cdot \boldsymbol{s}) \boldsymbol{m})(1-\cos \delta),
$$

with angle $\delta$ given by

$$
\sin \delta=\frac{(m+h) \sinh \zeta+(\boldsymbol{n} \cdot \boldsymbol{p})(\cosh \zeta-1)}{(m+h)\left(m+h^{\prime}\right)}|\boldsymbol{p} \times \boldsymbol{n}|,
$$

where $h^{\prime}=h \cosh \zeta+\boldsymbol{n} \cdot \boldsymbol{p} \sinh \zeta$ by Table 2 .

Under a boost in the direction of $\boldsymbol{n}$, the momentum also turns around $\boldsymbol{p} \times \boldsymbol{n}$. This is true in all generality: from the coadjoint action for boosts - see Table $2-$ with $p^{\prime}=\Lambda p$, we obtain

$$
\boldsymbol{p} \times \boldsymbol{p}^{\prime}=[h \sinh \zeta+(\boldsymbol{n} \cdot \boldsymbol{p})(\cosh \zeta-1)] \boldsymbol{p} \times \boldsymbol{n} .
$$


Therefore, the component of $\boldsymbol{p}^{\prime}$ not along $\boldsymbol{p}$ stays on the plane perpendicular to $\boldsymbol{p} \times \boldsymbol{n}$. The sine of the rotation angle is given by

$$
\frac{\left|\boldsymbol{p} \times \boldsymbol{p}^{\prime}\right|}{\left|\boldsymbol{p} \| \boldsymbol{p}^{\prime}\right|}=\frac{h \sinh \zeta+(\boldsymbol{n} \cdot \boldsymbol{p})(\cosh \zeta-1)}{|\boldsymbol{p}|\left|\boldsymbol{p}^{\prime}\right|}|\boldsymbol{p} \times \boldsymbol{n}| .
$$

This is in general greater than the Wigner angle given by (2.15). Now comes a key point: although not all the factors in its definition do so, the Wigner rotation formula itself makes perfect sense for $m=0$. Namely, keeping in mind that in this case $h=|\boldsymbol{p}|$ and $h^{\prime}=\left|\boldsymbol{p}^{\prime}\right|$, formula (2.15) then perfectly matches formula (2.16).

For good measure, we give next the brute-force proof that rotating $\boldsymbol{p}$ around $\boldsymbol{p} \times \boldsymbol{n}$ with rotation angle given by the massless limit of (2.15) yields the expected swing from a boost on $\boldsymbol{p}$. We shall also need that

$$
\sin \delta=\frac{h \sinh \zeta+(\boldsymbol{n} \cdot \boldsymbol{p})(\cosh \zeta-1)}{h h^{\prime}}|\boldsymbol{p} \times \boldsymbol{n}|, \quad \cos \delta=1-\frac{|\boldsymbol{p} \times \boldsymbol{n}|^{2}}{h h^{\prime}}(\cosh \zeta-1),
$$

in that limit. (Using $|\boldsymbol{p} \times \boldsymbol{n}|^{2}=h^{2}-(\boldsymbol{n} \cdot \boldsymbol{p})^{2}$, these expressions yield $\cos ^{2} \delta+\sin ^{2} \delta=1$, so they do define an angle $\delta$.) Now, since the axis of rotation $\boldsymbol{m}$ is perpendicular to $\boldsymbol{p}$, one finds from Eqn. (2.14) that

$$
\begin{aligned}
R_{\delta, \boldsymbol{m}}(\boldsymbol{p})= & \boldsymbol{p} \cos \delta+(\boldsymbol{m} \times \boldsymbol{p}) \sin \delta=\boldsymbol{p} \cos \delta+\left(h^{2} \boldsymbol{n}-(\boldsymbol{n} \cdot \boldsymbol{p}) \boldsymbol{p}\right) \frac{\sin \delta}{|\boldsymbol{p} \times \boldsymbol{n}|}, \\
= & \boldsymbol{p}-\left\{\frac{\cosh \zeta-1}{h h^{\prime}}\left(h^{2}-(\boldsymbol{n} \cdot \boldsymbol{p})^{2}\right)+\frac{\boldsymbol{n} \cdot \boldsymbol{p}}{h h^{\prime}}(h \sinh \zeta+\boldsymbol{n} \cdot \boldsymbol{p}(\cosh \zeta-1))\right\} \boldsymbol{p} \\
& \quad+\frac{h}{h^{\prime}}(h \sinh \zeta+\boldsymbol{n} \cdot \boldsymbol{p}(\cosh \zeta-1)) \boldsymbol{n} \\
= & \boldsymbol{p}-\frac{1}{h^{\prime}}(h(\cosh \zeta-1)+\boldsymbol{n} \cdot \boldsymbol{p} \sinh \zeta) \boldsymbol{p}+\frac{h}{h^{\prime}}(h \sinh \zeta+\boldsymbol{n} \cdot \boldsymbol{p}(\cosh \zeta-1)) \boldsymbol{n} \\
= & \boldsymbol{p}-\frac{h^{\prime}-h}{h^{\prime}} \boldsymbol{p}+\frac{h}{h^{\prime}}(h \sinh \zeta+\boldsymbol{n} \cdot \boldsymbol{p}(\cosh \zeta-1)) \boldsymbol{n} \\
= & \frac{h}{h^{\prime}}(\boldsymbol{p}+h \boldsymbol{n} \sinh \zeta+(\boldsymbol{n} \cdot \boldsymbol{p}) \boldsymbol{n}(\cosh \zeta-1))=\frac{h}{h^{\prime}} \boldsymbol{p}^{\prime} .
\end{aligned}
$$

Therefore, $\boldsymbol{p}^{\prime} / h^{\prime}=R_{\delta, \boldsymbol{m}}(\boldsymbol{p} / h)$, with $\boldsymbol{m}=(\boldsymbol{p} \times \boldsymbol{n}) /|\boldsymbol{p} \times \boldsymbol{n}|$ and $\delta$ given by (2.17).

\section{The shape of the orbits}

A nagging worry for some readers may have been that, contrary to the massive case, there is no distinguished point for the momentum in the orbit of a WP, nor there is a continuous cross-section of the Lorentz principal bundle over it [17]. Fortunately, the kinematics of the WP saves the day.

\subsection{Coordinate transformations}

In order to understand the structure of the orbits corresponding to Wigner particles, we need to examine the effect of boosts on $\lambda, \boldsymbol{t}, \boldsymbol{r}$, which perhaps is not obvious a priori. 
As previously indicated, the Coad formulas for $(h, \boldsymbol{p})$ are good for $\left(w^{0}, \boldsymbol{w}\right)$. With $\boldsymbol{n}$ the direction of a boost and $\zeta$ its parameter, we therefore obtain for the helicity (an invariant under translations and rotations):

$$
\lambda=\frac{w^{0}}{h} \longmapsto \frac{\left(w^{0}\right)^{\prime}}{h^{\prime}} \equiv \frac{w^{0} \cosh \zeta+\boldsymbol{n} \cdot \boldsymbol{w} \sinh \zeta}{h \cosh \zeta+\boldsymbol{n} \cdot \boldsymbol{p} \sinh \zeta}=\lambda+\frac{\boldsymbol{n} \cdot \boldsymbol{t} \tanh \zeta}{h+\boldsymbol{n} \cdot \boldsymbol{p} \tanh \zeta} .
$$

In particular $\lambda$ is invariant in the case $\boldsymbol{n}=\boldsymbol{p} / h$.

Next, $\boldsymbol{w}-w^{0} \boldsymbol{p} / h=\boldsymbol{t} \mapsto \boldsymbol{t}^{\prime}$, where

$$
\begin{aligned}
\boldsymbol{t}^{\prime} & :=\boldsymbol{t}+(\boldsymbol{n} \cdot \boldsymbol{t}) \boldsymbol{n}(\cosh \zeta-1)-\frac{\boldsymbol{n} \cdot \boldsymbol{t} \tanh \zeta}{h+\boldsymbol{n} \cdot \boldsymbol{p} \tanh \zeta}(\boldsymbol{p}+h \boldsymbol{n} \sinh \zeta+(\boldsymbol{n} \cdot \boldsymbol{p}) \boldsymbol{n}(\cosh \zeta-1)) \\
& =\boldsymbol{t}+\boldsymbol{n} \cdot \boldsymbol{t}\left((\cosh \zeta-1) \boldsymbol{n}-\sinh \zeta \boldsymbol{p}^{\prime} / h^{\prime}\right) .
\end{aligned}
$$

The expression (3.1) is linear in $\boldsymbol{t}$, with coefficients depending on $\boldsymbol{p}, \boldsymbol{n}, \zeta$. We verify it:

$$
\begin{aligned}
\boldsymbol{t}^{\prime}=\frac{h^{\prime} \boldsymbol{w}^{\prime}-\left(w^{0}\right)^{\prime} \boldsymbol{p}^{\prime}}{h^{\prime}}= & \frac{1}{h^{\prime}}\left[(h \cosh \zeta+\boldsymbol{n} \cdot \boldsymbol{p} \sinh \zeta)\left(\boldsymbol{w}+w^{0} \boldsymbol{n} \sinh \zeta+(\boldsymbol{n} \cdot \boldsymbol{w}) \boldsymbol{n}(\cosh \zeta-1)\right)\right. \\
& \left.-\left(w^{0} \cosh \zeta+\boldsymbol{n} \cdot \boldsymbol{w} \sinh \zeta\right)(\boldsymbol{p}+h \boldsymbol{n} \sinh \zeta+(\boldsymbol{n} \cdot \boldsymbol{p}) \boldsymbol{n}(\cosh \zeta-1))\right] .
\end{aligned}
$$

The computation proceeds by systematically cancelling all terms in $w^{0}$. There remains

$$
\begin{aligned}
\boldsymbol{t}^{\prime}= & \frac{1}{h^{\prime}}[h \cosh \zeta(\boldsymbol{t}+(\boldsymbol{n} \cdot \boldsymbol{t}) \boldsymbol{n}(\cosh \zeta-1))-(h(\boldsymbol{n} \cdot \boldsymbol{t}) \boldsymbol{n} \sinh \zeta-(\boldsymbol{n} \cdot \boldsymbol{p}) \boldsymbol{t}+(\boldsymbol{n} \cdot \boldsymbol{t}) \boldsymbol{p}) \sinh \zeta] \\
= & \boldsymbol{t}+(\boldsymbol{n} \cdot \boldsymbol{t}) \boldsymbol{n}(\cosh \zeta-1) \\
& \quad-\frac{1}{h^{\prime}}\left[h(\boldsymbol{n} \cdot \boldsymbol{t}) \boldsymbol{n} \sinh ^{2} \zeta+(\boldsymbol{n} \cdot \boldsymbol{t}) \boldsymbol{p} \sinh \zeta+(\boldsymbol{n} \cdot \boldsymbol{p})(\boldsymbol{n} \cdot \boldsymbol{t}) \boldsymbol{n} \sinh \zeta(\cosh \zeta-1)\right] \\
= & \boldsymbol{t}+(\boldsymbol{n} \cdot \boldsymbol{t}) \boldsymbol{n}(\cosh \zeta-1)-\frac{(\boldsymbol{n} \cdot \boldsymbol{t}) \sinh \zeta}{h^{\prime}}[\boldsymbol{p}+h \boldsymbol{n} \sinh \zeta+(\boldsymbol{n} \cdot \boldsymbol{p}) \boldsymbol{n}(\cosh \zeta-1)] . \\
= & \boldsymbol{t}+(\boldsymbol{n} \cdot \boldsymbol{t})\left[(\cosh \zeta-1) \boldsymbol{n}-\sinh \zeta \boldsymbol{p}^{\prime} / h^{\prime}\right] .
\end{aligned}
$$

Theorem 5. The mapping $\boldsymbol{t} \mapsto \boldsymbol{t}^{\prime}$ is implemented by the same rotation $R_{\delta, \boldsymbol{m}}$ of formula (2.18), with axis $\boldsymbol{m}:=(\boldsymbol{p} \times \boldsymbol{n}) /|\boldsymbol{p} \times \boldsymbol{n}|$ and angle $\delta$ given by (2.17). For $\boldsymbol{n}= \pm \boldsymbol{p} / h$, the rotation is trivial and $t^{\prime}=t$.

Proof. To check the equality $\boldsymbol{t}^{\prime}=R_{\delta, \boldsymbol{m}}(\boldsymbol{t})$, it is enough to show that these two 3-vectors have the same components with respect to some 3 -vector basis. For that purpose, choose the orthogonal moving frame $\{\boldsymbol{p} / h, \boldsymbol{t}, \boldsymbol{p} / h \times \boldsymbol{t}\}$. We claim that

$$
(p / h) \cdot t^{\prime}=(p / h) \cdot R_{\delta, m} t, \quad t \cdot t^{\prime}=t \cdot R_{\delta, m} t, \quad(p / h \times t) \cdot t^{\prime}=(p / h \times t) \cdot R_{\delta, m} t .
$$

First, of all,

$$
\begin{aligned}
(\boldsymbol{p} / h) \cdot \boldsymbol{t}^{\prime} & =(\boldsymbol{n} \cdot \boldsymbol{t})(\boldsymbol{n} \cdot \boldsymbol{p}) \frac{\cosh \zeta-1}{h}-\frac{(\boldsymbol{n} \cdot \boldsymbol{t}) \sinh \zeta}{h h^{\prime}}\left[h^{2}+h \boldsymbol{n} \cdot \boldsymbol{p} \sinh \zeta+(\boldsymbol{n} \cdot \boldsymbol{p})^{2}(\cosh \zeta-1)\right] \\
& =(\boldsymbol{n} \cdot \boldsymbol{t})(\boldsymbol{n} \cdot \boldsymbol{p}) \frac{\cosh \zeta-1}{h h^{\prime}}\left(h^{\prime}-h(\cosh \zeta+1)-\boldsymbol{n} \cdot \boldsymbol{p} \sinh \zeta\right)-\frac{h}{h^{\prime}} \boldsymbol{n} \cdot \boldsymbol{t} \sinh \zeta \\
& =-\frac{\boldsymbol{n} \cdot \boldsymbol{t}}{h^{\prime}}(h \sinh \zeta+\boldsymbol{n} \cdot \boldsymbol{p}(\cosh \zeta-1))=-\frac{h \boldsymbol{n} \cdot \boldsymbol{t}}{|\boldsymbol{p} \times \boldsymbol{n}|} \sin \delta \\
& =(\boldsymbol{p} / h) \cdot(\boldsymbol{m} \times \boldsymbol{t}) \sin \delta=(\boldsymbol{p} / h) \cdot \boldsymbol{R}_{\delta, \boldsymbol{m}} \boldsymbol{t} .
\end{aligned}
$$


Table 3: The coadjoint action on orbital coordinates

\begin{tabular}{|c|c|c|c|c|}
\hline$u \backslash X$ & $-a^{0} H$ & $a \cdot P$ & $\alpha \boldsymbol{m} \cdot \boldsymbol{L}$ & $\zeta n \cdot K$ \\
\hline$\lambda$ & $\lambda$ & $\lambda$ & $\lambda$ & $\lambda+\boldsymbol{n} \cdot \boldsymbol{t} \tanh \zeta /(h+\boldsymbol{n} \cdot \boldsymbol{p} \tanh \zeta)$ \\
\hline$p$ & $p$ & $p$ & $R_{\alpha, \boldsymbol{m}} \boldsymbol{p}$ & $\boldsymbol{p}+h \boldsymbol{n} \sinh \zeta+(\boldsymbol{n} \cdot \boldsymbol{p}) \boldsymbol{n}(\cosh \zeta-1)$ \\
\hline $\boldsymbol{r}$ & $\boldsymbol{r}-a^{0} \frac{\boldsymbol{p}}{h}$ & $r+a$ & $R_{\alpha, \boldsymbol{m}} \boldsymbol{r}$ & $\begin{aligned} \boldsymbol{r} & -\frac{\boldsymbol{n} \cdot \boldsymbol{r}}{h^{\prime}}(\boldsymbol{p} \sinh \zeta+h \boldsymbol{n}(\cosh \zeta-1)) \\
& +\frac{\lambda}{h h^{\prime}}(\boldsymbol{n} \times \boldsymbol{p}) \sinh \zeta+(\boldsymbol{t} \text {-dependent term })\end{aligned}$ \\
\hline$t$ & $t$ & $t$ & $R_{\alpha, \boldsymbol{m}} t$ & $R_{\delta, \boldsymbol{u}} \boldsymbol{t}$ (with $\delta$ as given in (2.17) and $\left.\boldsymbol{u}=\frac{\boldsymbol{p} \times \boldsymbol{n}}{|\boldsymbol{p} \times \boldsymbol{n}|}\right)$ \\
\hline
\end{tabular}

Next,

$$
\begin{aligned}
\boldsymbol{t} \cdot \boldsymbol{t}^{\prime} & =\rho^{2}+\frac{(\boldsymbol{n} \cdot \boldsymbol{t})^{2}}{h^{\prime}}\left[h^{\prime}(\cosh \zeta-1)-h \sinh ^{2} \zeta-(\boldsymbol{n} \cdot \boldsymbol{p}) \sinh \zeta(\cosh \zeta-1)\right] \\
& =\rho^{2}+\frac{(\boldsymbol{n} \cdot \boldsymbol{t})^{2}}{h^{\prime}}\left(h \cosh \zeta(\cosh \zeta-1)-h \sinh ^{2} \zeta\right)=\rho^{2}-\frac{h(\boldsymbol{n} \cdot \boldsymbol{t})^{2}}{h^{\prime}}(\cosh \zeta-1),
\end{aligned}
$$

whereas

$$
\begin{aligned}
\boldsymbol{t} \cdot R_{\delta, \boldsymbol{m}} \boldsymbol{t} & =\rho^{2}-\left(\rho^{2}-|\boldsymbol{m} \times \boldsymbol{t}|^{2}\right)(1-\cos \delta)=\rho^{2}-\frac{|(\boldsymbol{p} \times \boldsymbol{n}) \times \boldsymbol{t}|^{2}}{|\boldsymbol{p} \times \boldsymbol{n}|^{2}}(1-\cos \delta) \\
& =\rho^{2}-\frac{h(\boldsymbol{n} \cdot \boldsymbol{t})^{2}}{h^{\prime}}(\cosh \zeta-1),
\end{aligned}
$$

as claimed. We leave the proof of the third equation in (3.2) to the reader.

Consider boosts along the special directions $\boldsymbol{n}=\boldsymbol{p} / h, \boldsymbol{t} / \rho$ and $(\boldsymbol{p} \times \boldsymbol{t}) / h \rho$. (a) For $\boldsymbol{n}=\boldsymbol{p} / h$, we get $\boldsymbol{t}^{\prime}=\boldsymbol{t}$ and $\boldsymbol{p}^{\prime} / h^{\prime}=\boldsymbol{p} / h$ : trivial rotation. (b) For $\boldsymbol{n}=\boldsymbol{t} / \rho$, we get $h^{\prime}=h \cosh \zeta, \boldsymbol{m}=(\boldsymbol{p} \times \boldsymbol{t}) / h \rho$, and

$$
\boldsymbol{p}^{\prime} / h^{\prime}=(\boldsymbol{p} / h) \operatorname{sech} \zeta+(\boldsymbol{t} / \rho) \tanh \zeta, \quad \boldsymbol{t}^{\prime} / \rho=-(\boldsymbol{p} / h) \tanh \zeta+(\boldsymbol{t} / \rho) \operatorname{sech} \zeta .
$$

(c) For $\boldsymbol{n}=(\boldsymbol{p} \times \boldsymbol{t}) / h \rho$, again $h^{\prime}=h \cosh \zeta$ and $\boldsymbol{p}^{\prime} / h^{\prime}=(\boldsymbol{p} / h) \operatorname{sech} \zeta+(\boldsymbol{t} / \rho) \tanh \zeta$, but now $\boldsymbol{m}=-\boldsymbol{t} / \rho$ and $\boldsymbol{t}^{\prime}=\boldsymbol{t}$. In case (b) unsurprisingly there holds $\sin \delta=\tanh \zeta$ and $\cos \delta=\operatorname{sech} \zeta$.

Corollary 6. Under the Lorentz group action, the moving frame rotates as a gyroscope:

$$
p / h \mapsto R_{\delta, m}(p / h), \quad t \mapsto R_{\delta, m} t, \quad p / h \times t \mapsto R_{\delta, m}(p / h \times t),
$$

where $\boldsymbol{m}=(\boldsymbol{p} \times \boldsymbol{n}) /|\boldsymbol{p} \times \boldsymbol{n}|$.

Proof. The asserted result being true for all rotations and boosts, it is ipso facto true for all Lorentz transformations.

We deem quite noteworthy this remarkable kinematical behaviour of the WP. It has as a consequence Wigner's original equations of motion [18] in a first-quantized formulation [19]. 
We now examine the position coordinates: $-\boldsymbol{k} / h+(\boldsymbol{p} \times \boldsymbol{t}) / h^{3}=\boldsymbol{r} \mapsto \boldsymbol{r}^{\prime}$, where

$$
\begin{aligned}
\boldsymbol{r}^{\prime}:= & \frac{-\boldsymbol{k} \cosh \zeta+(\boldsymbol{n} \cdot \boldsymbol{k}) \boldsymbol{n}(\cosh \zeta-1)}{h^{\prime}}+\frac{\boldsymbol{n} \times \boldsymbol{l} \sinh \zeta}{h^{\prime}}+\frac{R_{\delta,(\boldsymbol{p} \times \boldsymbol{n}) /|\boldsymbol{p} \times \boldsymbol{n}|}(\boldsymbol{p} / h \times \boldsymbol{t})}{h^{\prime 2}} \\
= & \frac{h \boldsymbol{r} \cosh \zeta-h(\boldsymbol{n} \cdot \boldsymbol{r}) \boldsymbol{n}(\cosh \zeta-1)}{h^{\prime}}+\frac{((\boldsymbol{n} \cdot \boldsymbol{p}) \boldsymbol{r}-(\boldsymbol{n} \cdot \boldsymbol{r}) \boldsymbol{p}) \sinh \zeta}{h^{\prime}}+\frac{\lambda}{h h^{\prime}}(\boldsymbol{n} \times \boldsymbol{p}) \sinh \zeta \\
& -\frac{\boldsymbol{p} \times \boldsymbol{t} \cosh \zeta}{h^{2} h^{\prime}}+\frac{[\boldsymbol{n}, \boldsymbol{p}, \boldsymbol{t}] \boldsymbol{n}(\cosh \zeta-1)}{h^{2} h^{\prime}}+\frac{R_{\delta,(\boldsymbol{p} \times \boldsymbol{n}) /|\boldsymbol{p} \times \boldsymbol{n}|}(\boldsymbol{p} / h \times \boldsymbol{t})}{h^{\prime 2}} \\
= & \boldsymbol{r}-\frac{\boldsymbol{n} \cdot \boldsymbol{r}}{h^{\prime}}(\boldsymbol{p} \sinh \zeta+h \boldsymbol{n}(\cosh \zeta-1))+(\lambda, \boldsymbol{t}) \text {-dependent terms. }
\end{aligned}
$$

The first three terms in (3.3), free of the internal variables $\lambda, t$, look different from the transformation rule for momentum; however, we shall soon see that they make relativistic sense.

The action of the Poincaré group generators now follows from (3.1) and (3.3). They are given in Table 3.

For good measure, the infinitesimal actions are also given in Table $4 .^{6}$ It helps to note that this action on $w$ follows the pattern of its action on $p$ : namely, $(\zeta \boldsymbol{n} \cdot \boldsymbol{K}) \triangleright h=\zeta \boldsymbol{n} \cdot \boldsymbol{p}$ whereas $(\zeta \boldsymbol{n} \cdot \boldsymbol{K}) \triangleright w^{0}=\zeta \boldsymbol{n} \cdot \boldsymbol{w} ;(\alpha \boldsymbol{m} \cdot \boldsymbol{L}) \triangleright w^{0}=0 ;(\zeta \boldsymbol{n} \cdot \boldsymbol{K}) \triangleright \boldsymbol{w}=w^{0} \zeta \boldsymbol{n}$ and $(\alpha \boldsymbol{m} \cdot \boldsymbol{L}) \triangleright \boldsymbol{w}=\alpha \boldsymbol{m} \times \boldsymbol{w}$; and the other generators act trivially on $w^{0}$ and $\boldsymbol{w}$.

The $(3,4)$-entry in Table 4 is found by expanding the right hand side of $(3.3)$ in powers of $\zeta$, using $\sinh \zeta=O(\zeta), \cosh \zeta-1=O\left(\zeta^{2}\right), \cos \delta=1+O\left(\zeta^{2}\right),(\boldsymbol{p} \times \boldsymbol{n}) \sinh \delta /|\boldsymbol{p} \times \boldsymbol{n}|=-\zeta \boldsymbol{n} \times \boldsymbol{p} / h+O\left(\zeta^{2}\right)$, and $h / h^{\prime}=1-\zeta \boldsymbol{n} \cdot \boldsymbol{p} / h+O\left(\zeta^{2}\right)$. Therefore:

$$
\begin{aligned}
\boldsymbol{r}^{\prime}= & \boldsymbol{r}-(\zeta \boldsymbol{n} \cdot \boldsymbol{p} / h) \boldsymbol{r}+\frac{(\zeta \boldsymbol{n} \cdot \boldsymbol{p}) \boldsymbol{r}-(\zeta \boldsymbol{n} \cdot \boldsymbol{r}) \boldsymbol{p}}{h}+\frac{\lambda}{h^{2}} \zeta \boldsymbol{n} \times \boldsymbol{p}-\frac{\boldsymbol{p} \times \boldsymbol{t}}{h^{3}}+\frac{(\zeta \boldsymbol{n} \cdot \boldsymbol{p}) \boldsymbol{p} \times \boldsymbol{t}}{h^{4}} \\
& +\frac{\boldsymbol{p} / h \times \boldsymbol{t}}{h^{2}}-2(\zeta \boldsymbol{n} \cdot \boldsymbol{p} / h) \frac{\boldsymbol{p} / h \times \boldsymbol{t}}{h^{2}}-\frac{(\zeta \boldsymbol{n} \times \boldsymbol{p} / h) \times(\boldsymbol{p} / h \times \boldsymbol{t})}{h^{2}}+O\left(\zeta^{2}\right) \\
= & \boldsymbol{r}-(\zeta \boldsymbol{n} \cdot \boldsymbol{r}) \boldsymbol{p} / h+\lambda \zeta \boldsymbol{n} \times \boldsymbol{p} / h^{2}-\frac{(\zeta \boldsymbol{n} \cdot \boldsymbol{p}) \boldsymbol{p} \times \boldsymbol{t}+\zeta \boldsymbol{n} \cdot(\boldsymbol{p} \times \boldsymbol{t}) \boldsymbol{p}}{h^{4}}+O\left(\zeta^{2}\right) \\
= & \boldsymbol{r}-(\zeta \boldsymbol{n} \cdot \boldsymbol{r}) \boldsymbol{p} / h+\lambda \zeta \boldsymbol{n} \times \boldsymbol{p} / h^{2}-\zeta \boldsymbol{n} \times((\boldsymbol{p} \times \boldsymbol{t}) \times \boldsymbol{p}) / h^{4}-2 \zeta \boldsymbol{n} \cdot(\boldsymbol{p} \times \boldsymbol{t}) \boldsymbol{p} / h^{4}+O\left(\zeta^{2}\right) \\
= & \boldsymbol{r}-(\zeta \boldsymbol{n} \cdot \boldsymbol{r}) \boldsymbol{p} / h+\zeta \boldsymbol{n} \times(\lambda \boldsymbol{p}-\boldsymbol{t}) / h^{2}-2 \zeta \boldsymbol{n} \cdot(\boldsymbol{p} \times \boldsymbol{t}) \boldsymbol{p} / h^{4}+O\left(\zeta^{2}\right) .
\end{aligned}
$$

The shape of the orbit can be determined already: clearly $\boldsymbol{r}$ takes values in $\mathbb{R}^{3}$, then $\boldsymbol{p}$ takes values in $\mathbb{R}^{3} \backslash\{\boldsymbol{0}\} \approx \mathbb{R} \times \mathbb{S}^{2}$. Then $\lambda \in(-\infty, \infty)$ and $\boldsymbol{t}$ takes values on a circle. Therefore ${ }^{7}$

$$
\mathcal{O}_{m=0, \rho} \approx \mathbb{R}^{3} \times\left(\mathbb{R} \times \mathbb{S}^{2}\right) \times\left(\mathbb{R} \times \mathbb{S}^{1}\right) .
$$

\subsubsection{On the stability subgroup}

Choose any point $u=(\lambda, \boldsymbol{p}, \boldsymbol{r}, \boldsymbol{t})$ in $\mathfrak{p}^{*}$ subject to the requirements $|\boldsymbol{p}|^{2}=h^{2}$ and $|\boldsymbol{t}|^{2}=\rho^{2}>0$. To study its coadjoint orbit under the Poincaré group, it is also instructive to determine the isotropy subgroup $\mathcal{P}_{u}$, since the orbit is just the homogeneous space $\mathcal{P} / \mathcal{P}_{u}$.

${ }^{6}$ The information is already contained in the Poisson brackets, but it is good to cross-check them with the outcomes in Table 3.

7There is now a coadjoint orbit that is not simply connected. For non-simply connected coadjoint orbits it is hard to push forward the Kirillov paradigm - the known examples such as [20] correspond to groups with trivial stability subgroups - towards the derivation of the unitary irreducible representations of the group. 
Table 4: The infinitesimal coadjoint action $X \triangleright u=\operatorname{coad}(X) u$

\begin{tabular}{c|cccl}
\hline \hline$u \backslash X$ & $-a^{0} H$ & $\boldsymbol{a} \cdot \boldsymbol{P}$ & $\alpha \boldsymbol{m} \cdot \boldsymbol{L}$ & $\zeta \boldsymbol{n} \cdot \boldsymbol{K}$ \\
\hline$\lambda$ & 0 & 0 & 0 & $\zeta \boldsymbol{n} \cdot \boldsymbol{t} / h$ \\
$\boldsymbol{p}$ & $\mathbf{0}$ & $\mathbf{0}$ & $\alpha \boldsymbol{m} \times \boldsymbol{p}$ & $h \zeta \boldsymbol{n}$ \\
$\boldsymbol{r}$ & $-a^{0} \boldsymbol{p} / h$ & $\boldsymbol{a}$ & $\alpha \boldsymbol{m} \times \boldsymbol{r}$ & $-(\zeta \boldsymbol{n} \cdot \boldsymbol{r}) \boldsymbol{p} / h+\zeta \boldsymbol{n} \times(\lambda \boldsymbol{p}-\boldsymbol{t}) / h^{2}-2 \zeta \boldsymbol{n} \cdot(\boldsymbol{p} \times \boldsymbol{t}) \boldsymbol{p} / h^{4}$ \\
$\boldsymbol{t}$ & $\mathbf{0}$ & $\mathbf{0}$ & $\alpha \boldsymbol{m} \times \boldsymbol{t}$ & $-(\zeta \boldsymbol{n} \cdot \boldsymbol{t}) \boldsymbol{p} / h$ \\
\hline \hline
\end{tabular}

The isotropy subgroups at different points on the orbit are conjugate, so to find a "representative" isotropy group we choose a point where $\boldsymbol{r}=\mathbf{0}$ and $\lambda=0$. We tackle Table 3 one row at a time. From invariance of $\lambda$, excluding the case $\zeta=0$, we get $\boldsymbol{n} \cdot \boldsymbol{t}=0$ for the boost component of an element of the stability subgroup. Therefore

$$
\boldsymbol{n}=a \boldsymbol{p} / h+b(\boldsymbol{p} \times \boldsymbol{t}) / \rho h, \text { with } a^{2}+b^{2}=1,
$$

and $\boldsymbol{u} \equiv(\boldsymbol{p} \times \boldsymbol{n}) /|\boldsymbol{p} \times \boldsymbol{n}|=\boldsymbol{t} / \rho$. Thus a boost leaving $\lambda$ invariant will leave $\boldsymbol{t}$ invariant as well. That is indeed so, since then $R_{\delta, u}$ is a rotation around the direction of $t$ itself. The $R_{\alpha, m}$ component of that element fixes $\boldsymbol{t}$, too, so $\boldsymbol{m}=\boldsymbol{u}$. From the second row, we learn that $a$ in equation (3.5) equals $(1-\cosh \zeta) / \sinh \zeta$ (necessarily $<0$ ), in order to keep $|\boldsymbol{p}|$ constant, whereby $h^{\prime}=h$ in this case. Since $\boldsymbol{p}$ rotates around $\boldsymbol{t}$, this can be compensated by an ordinary rotation around the same axis, with the same rotation angle in the opposite direction (i.e., $\alpha \boldsymbol{m}=-\delta \boldsymbol{u}$ ). Finally, from the third row, the nonvanishing terms of a boost action on $\boldsymbol{r}=\mathbf{0}$ and $\lambda=0$ produce components along $\boldsymbol{p}$ and $\boldsymbol{p} \times \boldsymbol{t}$, which can be compensated by a one-dimensional family of choices of $a^{0}$, and a suitable choice of $\boldsymbol{a}(\zeta)$ - we need not give its complicated formula here. Notice that in the present instance no purely-Lorentz solutions can be found. ${ }^{8}$ The isotropy subgroup, freely parametrized by $a^{0}$ and $\zeta$, has the topology of the plane $\mathbb{R}^{2}$.

\subsubsection{Simultaneity hyperplanes}

The $r^{a}$ coordinates are not canonical, since they do not commute among themselves. We next examine whether they are relativistically covariant. Certainly they are covariant under Euclidean transformations. The argument that follows, taken from [21, Ch. 20], allows to understand the rule of change when going from one Lorentz frame to another (say with primed coordinates) by a boost. In a Hamiltonian formulation, the position coordinates should be regarded as initial conditions for free motion. Thus consider $\boldsymbol{r}(t)=\boldsymbol{r}+(\boldsymbol{p} / h) t$ and likewise $\boldsymbol{r}^{\prime}\left(t^{\prime}\right)=\boldsymbol{r}^{\prime}+\left(\boldsymbol{p}^{\prime} / h^{\prime}\right) t^{\prime}$, and assume the standard transformation rules under boosts:

$$
t^{\prime}=t \cosh \zeta+\boldsymbol{n} \cdot \boldsymbol{r}(t) \sinh \zeta ; \quad \boldsymbol{r}^{\prime}\left(t^{\prime}\right)=\boldsymbol{r}(t)+t \boldsymbol{n} \sinh \zeta+(\boldsymbol{n} \cdot \boldsymbol{r}(t)) \boldsymbol{n}(\cosh \zeta-1) .
$$

We want to examine the resulting relation between $\boldsymbol{r}^{\prime}\left(t^{\prime}=0\right) \equiv \boldsymbol{r}^{\prime}$ and $\boldsymbol{r}(t=0) \equiv \boldsymbol{r}$. Let us set $t^{\prime}=0$, obtaining

$$
t=-(\boldsymbol{n} \cdot \boldsymbol{r}) \frac{h \sinh \zeta}{h^{\prime}} \quad \text { and } \quad \boldsymbol{r}(t)=\boldsymbol{r}-(\boldsymbol{n} \cdot \boldsymbol{r}) \frac{\boldsymbol{p} \sinh \zeta}{h^{\prime}} .
$$

${ }^{8}$ There is a one-dimensional subspace of translations that acts trivially: when $\zeta=0$, one can take $\alpha=0$ as well, and Table 3 shows that the condition $\boldsymbol{a}=a^{0}(\boldsymbol{p} / h)$ yields invariance of $u$ under the coadjoint action. This is identical to what would occur for massive particles. 
Then in (3.6) the second equation becomes:

$$
\begin{aligned}
\boldsymbol{r}^{\prime} & =\boldsymbol{r}-(\boldsymbol{n} \cdot \boldsymbol{r}) \boldsymbol{p} \frac{\sinh \zeta}{h^{\prime}}+(\boldsymbol{n} \cdot \boldsymbol{r}) \boldsymbol{n}\left((\cosh \zeta-1)\left(1-(\boldsymbol{n} \cdot \boldsymbol{p}) \sinh \zeta / h^{\prime}\right)-h \sinh ^{2} \zeta / h^{\prime}\right) \\
& =\boldsymbol{r}-(\boldsymbol{n} \cdot \boldsymbol{r}) \boldsymbol{p} \frac{\sinh \zeta}{h^{\prime}}+(\boldsymbol{n} \cdot \boldsymbol{r}) \boldsymbol{n} \frac{h}{h^{\prime}}\left(\cosh \zeta(\cosh \zeta-1)-\sinh ^{2} \zeta\right) \\
& =\boldsymbol{r}-(\boldsymbol{n} \cdot \boldsymbol{r})(\boldsymbol{p} \sinh \zeta+h \boldsymbol{n}(\cosh \zeta-1)) / h^{\prime},
\end{aligned}
$$

reproducing the external coordinate part of the rule (3.3), on the nose. In conclusion, the first three terms are the expected ones for a structureless particle. Such an expression as above does not relate two coordinatizations of the same set of events, but the values of the position coordinates at the simultaneity hyperplanes $t=0$ and $t^{\prime}=0$ of the two frames related by the boost. It renders the position coordinates' Lorentz transformation behaviour in a formulation in which time has been eliminated: that is to say, the transformations are regarded as acting on the initial conditions (points of the coadjoint orbit) of a covariant formulation - see [3, Sect. 3] as well as the discussion in [21, Ch. 20].

In spite of the above, the $\boldsymbol{r}$-position coordinates are not relativistically covariant, due to the internal variables. When $\lambda$ is just a parameter and $\boldsymbol{t}$ is set to $\mathbf{0}$, we are in the fixed-helicity context; and that is still the case. The phenomenon is not new: for the massive particles with spin, one can find both (global) canonical and covariant position coordinates; but they do not coincide. This has been known for a good while [22]. The limit of the covariant coordinates $\boldsymbol{x}$ in the massive case,

$$
\boldsymbol{x}:=-\frac{\boldsymbol{k}}{h}-\frac{\boldsymbol{p} \times \boldsymbol{s}}{m h}=-\frac{\boldsymbol{k}}{h}-\frac{\boldsymbol{p} \times \boldsymbol{w}}{m^{2} h},
$$

as $m \downarrow 0, s \uparrow \infty$ is singular, at any rate. Local canonical coordinates always exist, due to Darboux's theorem - see the next subsection 3.2. It is an open question whether covariant coordinates exist in our case; experience [3] suggests that it would be rewarding to work with them.

\subsection{Slant and the symplectic structure}

Since $\boldsymbol{t}$ takes values on a circle, it is most natural to regard it as being given by an angle $\theta$, as is done in [23] for a quantum counterpart. Let $\boldsymbol{t}_{1}(\boldsymbol{p}), \boldsymbol{t}_{2}(\boldsymbol{p}) \perp \boldsymbol{p}$, with moreover $\boldsymbol{t}_{1}(\boldsymbol{p}) \perp \boldsymbol{t}_{2}(\boldsymbol{p})$, be chosen spacelike vectors of length $\rho$; and let us expand $t$ in terms of these:

$$
\boldsymbol{t}=\alpha_{1} \boldsymbol{t}_{1}(\boldsymbol{p})+\alpha_{2} \boldsymbol{t}_{2}(\boldsymbol{p}) .
$$

Then $\left|\alpha_{1}\right|^{2}+\left|\alpha_{2}\right|^{2}=1$, and we express $t$ through $\alpha_{1}=\cos \theta, \alpha_{2}=\sin \theta$. Such an internal variable $\theta$ we baptize here the slant. The decomposition is clearly non-unique, so some choices need to be made. Since $\boldsymbol{p}$ and $\lambda$ Poisson-commute, it is natural to ask that $\left\{\lambda, t_{1}(\boldsymbol{p})\right\}=0$. In this way,

$$
\begin{aligned}
\alpha_{1}\left(\boldsymbol{p} / h \times \boldsymbol{t}_{1}(\boldsymbol{p})\right)+\alpha_{2}\left(\boldsymbol{p} / h \times \boldsymbol{t}_{2}(\boldsymbol{p})\right) & =\boldsymbol{p} / h \times \boldsymbol{t} \\
= & -\{\lambda, \boldsymbol{t}\}=-\{\lambda, \cos \theta\} \boldsymbol{t}_{1}(\boldsymbol{p})-\{\lambda, \sin \theta\} \boldsymbol{t}_{2}(\boldsymbol{p})-\sin \theta\left\{\lambda, \boldsymbol{t}_{2}(\boldsymbol{p})\right\} .
\end{aligned}
$$

This leads us to choose $\boldsymbol{t}_{2}(\boldsymbol{p})=\boldsymbol{p} / h \times \boldsymbol{t}_{1}(\boldsymbol{p})$, therefore $\left\{\lambda, \boldsymbol{t}_{2}(\boldsymbol{p})\right\}=0$ and $\boldsymbol{t}_{1}(\boldsymbol{p})=-\boldsymbol{p} / h \times \boldsymbol{t}_{2}(\boldsymbol{p})$, so that

$$
\boldsymbol{p} / h \times \boldsymbol{t}=\boldsymbol{t}_{2}(\boldsymbol{p}) \cos \theta-\boldsymbol{t}_{1}(\boldsymbol{p}) \sin \theta \quad \text { and } \quad\{\lambda, \cos \theta\}=\sin \theta, \quad\{\lambda, \sin \theta\}=-\cos \theta,
$$


that is, $\left\{\lambda, e^{i \theta}\right\}=-i e^{i \theta}$. In conclusion, $\lambda$ and $\theta$ are symplectically conjugate variables.

Now consider the Poisson brackets involving components of $\boldsymbol{r}$ and $\boldsymbol{t}$. Given that $\{\boldsymbol{r}, \lambda\}=\mathbf{0}$, the natural choice is to take $\left\{r^{a}, \cos \theta\right\}=\left\{r^{a}, \sin \theta\right\}=0$, so that the spatial and internal coordinates symplectically decouple completely. This is consistent: the only check that we have on our choices so far is that $\left\{r^{a}, \boldsymbol{t}\right\}=-t^{a} \boldsymbol{p} / h^{2}$, from (2.13). This can be satisfied on deciding for

$$
\begin{aligned}
\left\{r^{a}, \boldsymbol{t}_{1}(\boldsymbol{p})\right\} & =-\left(t^{a} \cos \theta / h^{2}+(\boldsymbol{p} \times \boldsymbol{t})^{a} \sin \theta / h^{3}\right) \boldsymbol{p}, \quad \text { implying } \\
\left\{r^{a}, \boldsymbol{t}_{2}(\boldsymbol{p})\right\} & =-\left(t^{a} \sin \theta / h^{2}-(\boldsymbol{p} \times \boldsymbol{t})^{a} \cos \theta / h^{3}\right) \boldsymbol{p} ; \quad \text { and } \\
\left\{r^{a}, \boldsymbol{t}\right\} & =-\left(\cos ^{2} \theta+\sin ^{2} \theta\right) t^{a} \boldsymbol{p} / h^{2} .
\end{aligned}
$$

It should be remarked that the moving-frame component of $\boldsymbol{r}$ along $\boldsymbol{t}$ alone does not Poissoncommute with $\boldsymbol{t}$ :

$$
\{\boldsymbol{p} \cdot \boldsymbol{r}, \boldsymbol{t}\}=\mathbf{0}=\{[\boldsymbol{p}, \boldsymbol{t}, \boldsymbol{r}], \boldsymbol{t}\} ; \quad\{\boldsymbol{t} \cdot \boldsymbol{r}, \boldsymbol{t}\}=-\left(\rho^{2} / h^{2}\right) \boldsymbol{p} .
$$

Schwinger points in [15] to the duality of the spinning massless relativistic problem with that of an electrically charged particle in the distant field of a stationary magnetic charge. This was further explored by Bacry [24]. Indeed, in view of Lemma 4, the spatial component of the Poisson brackets for our problem in the $\left(r^{a}, p^{b}\right)$ coordinates is of the form

$$
\{f, g\}(\boldsymbol{r}, \boldsymbol{p})=\frac{\partial f}{\partial r^{a}} \frac{\partial g}{\partial p_{a}}-\frac{\partial f}{\partial p_{a}} \frac{\partial g}{\partial r^{a}}-\varepsilon^{a b c} \lambda \frac{p_{c}}{h^{3}} \frac{\partial f}{\partial r^{a}} \frac{\partial g}{\partial r^{b}}
$$

where in the fixed-helicity case $\lambda$ is replaced by a number. ${ }^{9}$ We have arrived at a Poisson matrix of the form

$$
\left(\begin{array}{cccccccc}
0 & 1 & -\lambda p_{3} / h^{3} & 0 & \lambda p_{2} / h^{3} & 0 & 0 & 0 \\
-1 & 0 & 0 & 0 & 0 & 0 & 0 & 0 \\
\lambda p_{3} / h^{3} & 0 & 0 & 1 & -\lambda p_{1} / h^{3} & 0 & 0 & 0 \\
0 & 0 & -1 & 0 & 0 & 0 & 0 & 0 \\
-\lambda p_{2} / h^{3} & 0 & \lambda p_{1} / h^{3} & 0 & 0 & 1 & 0 & 0 \\
0 & 0 & 0 & 0 & -1 & 0 & 0 & 0 \\
0 & 0 & 0 & 0 & 0 & 0 & 0 & 1 \\
0 & 0 & 0 & 0 & 0 & 0 & -1 & 0
\end{array}\right)
$$

and the helicity $\lambda$ plays a role dual to a magnetic monopole charge. That is to say, the symplectic form on the WP orbits is given by

$$
\omega=d r^{a} \wedge d p_{a}-\varepsilon_{a b}^{c} \lambda p_{c} h^{-3} d r^{a} \wedge d r^{b}+d \lambda \wedge d \theta
$$

Thus we have obtained a Schwinger-Bacry structure, by now well known [25-27]. A quaternionic setting for it was devised by Emch and Jadczyk in [28], further explored by Cariñena, Marmo and three of us in $[29,30]$ and by Soloviev in $[26,27]$. It would be most interesting to know whether it is relevant for the Wigner particle. ${ }^{10}$

\footnotetext{
${ }^{9}$ Since the $\boldsymbol{r}$ and $\boldsymbol{p}$-variables are conjugate, by Lemma 4 , in the rest of this section we shall write $p_{b}$ rather than $p^{b}$ for the components of $\boldsymbol{p}$, as is customary.

${ }^{10}$ The simpler ordinary "magnetic" Poisson bracket has been the object of several studies leading up to an (already rather inexplicit) magnetic Weyl-Moyal product [31,32].
} 
Since the "magnetic terms" drop out from $\omega^{\wedge 4}$, a Liouville measure on the orbit is immediately seen to be

$$
\mu:=\omega^{\wedge 4} \propto d r^{1} \wedge d r^{2} \wedge d r^{3} \wedge d p_{3} \wedge d p_{2} \wedge d p_{1} \wedge d \lambda \wedge d \theta
$$

By general theory, Darboux or canonical coordinates $q^{a}$ do always exist locally [33]. To find them in our case, the systematic method advocated in [34] is not necessary. For the $6 \times 6$ principal submatrix here they are computed by the standard physical procedure of defining a "vector potential" $\boldsymbol{V}(\boldsymbol{p})$ such that

$$
\partial_{b} V_{a}-\partial_{a} V_{b}=-\varepsilon_{a b}^{c} \lambda p_{c} h^{-3}=\varepsilon_{a b}^{c} \lambda \partial_{c}\left(h^{-1}\right) .
$$

Then $q^{a}:=r^{a}+V^{a}$ foots the bill. An example is: $\boldsymbol{V}(\boldsymbol{p})=\left(p_{2},-p_{1}, 0\right) /|\boldsymbol{p}|\left(p_{3}+|\boldsymbol{p}|\right) .{ }^{11}$

\section{Conclusion and outlook}

The renewed excitement on the Wigner particles is a refreshing novelty in what seemed a foreclosed issue: the identification of physical particles. Although it is true that the massless WPs analyzed in this paper are not established members of the present zoo of particles, the fact that they have been relatively little studied leaves the possibility of important surprises wide open. While the final aim is a consistent quantum field theory [12], the Kirillov orbit method we have described here is a prelude to a full-fledged first quantization of these particles.

The nonzero Poisson bracket among the coordinates (2.13) also makes the configuration space a "noncommutative geometry". One further step in that direction would be to proceed along the lines of deformation quantization, and present a Groenewold-Moyal star product, like the ones obtained in [3]. This could be done with a generalization of the invertible Wigner transform to this case; it is a nontrivial task. It is likely that the definition of a product to low orders in $\hbar$ à la Kontsevich may be more tractable. We recall that a similar problem is found for the quantization of particles in a magnetic monopole background [25-30]. A related issue is the existence of covariant coordinates, and their eventual role in a quantization procedure.

Finally: with a few exceptions [36,37], so far most of the studies of WPs have been made for the bosonic case. Relatively little is known about them in the spin case, whose quantization may be of interest.

\section{A The Poincaré Lie algebra}

We briefly summarize our notational conventions for the generators of the Poincare group.

We use the Minkowski metric whose inner product of 4-vectors $x=\left(x^{0}, \boldsymbol{x}\right), y=\left(y^{0}, \boldsymbol{y}\right)$ is denoted by parentheses: $(x y)=x^{\mu} y_{\mu}:=x^{0} y^{0}-\boldsymbol{x} \cdot \boldsymbol{y}$. As usual, we write $x^{2}=(x x)=x^{\mu} x_{\mu}$.

The (restricted) Poincaré group $\mathcal{P}$ is the semidirect product $T_{4} \rtimes \mathcal{L}_{+}^{\uparrow}$, with multiplication written as $(a, \Lambda) \cdot\left(a^{\prime}, \Lambda^{\prime}\right)=\left(a+\Lambda a^{\prime}, \Lambda \Lambda^{\prime}\right)$. Its Lie algebra $\mathfrak{p}$ has a basis $\left\{H, P^{a}, L^{a}, K^{a}: a=1,2,3\right\}$, corresponding respectively to time translations, space translations, rotations and boosts. The

\footnotetext{
${ }^{11}$ From this (or a similar) formula it should be clear that under quantization one expects functional-analytic complications in the ket space - of the kind discussed in [35].
} 
nonzero commutation relations are as follows:

$$
\begin{aligned}
& {\left[L^{a}, L^{b}\right]=\varepsilon^{a b}{ }_{c} L^{c}, \quad\left[L^{a}, K^{b}\right]=\varepsilon^{a b}{ }_{c} K^{c}, \quad\left[K^{a}, K^{b}\right]=-\varepsilon^{a b}{ }_{c} L^{c},} \\
& {\left[L^{a}, P^{b}\right]=\varepsilon^{a b}{ }_{c} P^{c}, \quad\left[P^{b}, K^{a}\right]=\delta^{a b} H, \quad\left[H, K^{a}\right]=P^{a} \text {. }}
\end{aligned}
$$

The Lorentz-subgroup generators are also denoted by $J^{0 a}:=K^{a}, J^{a b}=\varepsilon^{a b}{ }_{c} L^{c}$. Note as well that $J_{0 a}=-J^{0 a}=-K^{a}, J_{a b}=J^{a b}=\varepsilon_{a b c} L^{c}$, and both $J^{\mu \nu}$ and $J_{\rho \sigma}$ are skewsymmetric tensors. The commutation relation of the latter may be summarized as

$$
\left[J_{\rho \sigma}, J_{\mu \nu}\right]=-g_{\rho \mu} J_{\sigma v}-g_{\sigma v} J_{\rho \mu}+g_{\sigma \mu} J_{\rho v}+g_{\rho v} J_{\sigma \mu} .
$$

The dual tensor $J^{* \rho \mu}:=-\frac{1}{2} \varepsilon^{\rho \mu \nu \tau} J_{v \tau}$ plays a role in the theory of the WP. Here $J^{* 0 a}=-L^{a}$ and $J^{* a b}=\varepsilon^{a b}{ }_{c} K^{c}$. Remark that

$$
\boldsymbol{K} \cdot \boldsymbol{L}=\frac{1}{2} J_{\rho \mu} J^{* \rho \mu} \quad \text { and } \quad \boldsymbol{K}^{2}-\boldsymbol{L}^{2}=\frac{1}{2} J_{\rho \mu} J^{\rho \mu}=-\frac{1}{2} J_{\rho \mu}^{*} J^{* \rho \mu}
$$

are the Casimirs of the Lorentz group.

\section{B A pedestrian approach to the "Pauli-Lubański limit"}

In our classical context, it is possible to obtain the WP data by carefully taking the $m \downarrow 0, s \uparrow \infty$ limit (with $m s$ finite). This we do essentially following Schwinger, mutatis mutandis. To the purpose, let us go back to equation (2.6), where it is clear that

$$
\lim _{m \downarrow 0, s \uparrow \infty} m \boldsymbol{s}=\boldsymbol{t} \perp \boldsymbol{p} .
$$

Clearly as well, from $\left\{s^{a}, s^{b}\right\}=\varepsilon^{a b}{ }_{c} s^{c}$ we infer $\left\{t^{a}, t^{b}\right\}=0$ and $\{\lambda, \boldsymbol{t}\}=-\boldsymbol{p} / h \times \boldsymbol{t}$, as well as the other brackets in Lemma 3. The interesting part is that instead of $\boldsymbol{q}$ as given in (2.7), which is ill-defined in the $m \downarrow 0, s \uparrow \infty$ limit, one can define already an $\boldsymbol{r}$-vector in the massive case enjoying a smooth Pauli-Lubański limit by

$$
\boldsymbol{r}:=\boldsymbol{q}+\frac{\boldsymbol{p} \times \boldsymbol{s}}{h^{2}}=-\frac{\boldsymbol{k}}{h}-\frac{\boldsymbol{p} \times \boldsymbol{s}}{h(m+h)}+\frac{\boldsymbol{p} \times \boldsymbol{s}}{h^{2}}=-\frac{\boldsymbol{k}}{h}+\frac{\boldsymbol{p} \times m \boldsymbol{s}}{h^{2}(m+h)}
$$

and already (2.11) holds, too, as well as the "magnetic" commutation relations between the $r^{a}$ given in (2.13):

$$
\begin{aligned}
\left\{r^{a}, r^{b}\right\} & =\left\{q^{a}, \varepsilon^{b}{ }_{e f} p^{e} s^{f} h^{-2}\right\}+\left\{\varepsilon^{a}{ }_{c d} p^{c} s^{d} h^{-2}, q^{b}\right\}+\varepsilon^{a}{ }_{c d} \varepsilon^{b}{ }_{e f} p^{c} p^{e}\left\{s^{d}, s^{f}\right\} h^{-4} \\
& =-2 \varepsilon^{a b}{ }_{c} s^{c} h^{-2}-2 \varepsilon^{b}{ }_{c d} p^{a} p^{c} s^{d} h^{-4}+2 \varepsilon^{a}{ }_{c d} p^{b} p^{c} s^{d} h^{-4}+\varepsilon^{a}{ }_{c d} \varepsilon^{b}{ }_{e f} \varepsilon^{d f}{ }_{g} p^{c} p^{e} s^{g} h^{-4} \\
& =-2 \varepsilon^{a b}{ }_{c} s^{c} h^{-2}-2 p^{a}(\boldsymbol{p} \times s)^{b} h^{-4}+2 p^{b}(\boldsymbol{p} \times s)^{a} h^{-4}+\varepsilon^{a b}{ }_{c} p^{c} w^{0} h^{-4},
\end{aligned}
$$

on using $w^{0}=\boldsymbol{p} \cdot \boldsymbol{s}$ in the massive case. Since $\boldsymbol{p} \times(\boldsymbol{p} \times \boldsymbol{s})=w^{0} \boldsymbol{p}-h^{2} \boldsymbol{s}$, this reduces to

$$
\left\{r^{a}, r^{b}\right\}=\varepsilon^{a b}{ }_{c}\left(-2 s^{c} h^{-2}-2\left(w^{0} p^{c}-h^{2} s^{c}\right) h^{-4}+w^{0} p^{c} h^{-4}\right)=-\varepsilon^{a b}{ }_{c} w^{0} p^{c} h^{-4}=-\varepsilon^{a b}{ }_{c} \lambda p^{c} h^{-3},
$$

in agreement with the last formula of Lemma 4. 


\section{Acknowledgements}

We are grateful for discussions with Alejandro Jenkins - and his astute comments regarding the gyroscope. We thank the referee for a careful reading and for suggesting welcome clarifications. JMG-B and JCV heartily thank the Università di Napoli Federico II and INFN Sezione di Napoli for its hospitality, and JMG-B wishes also to thank the ZiF at the University of Bielefeld for its warm welcome at the final stage of the paper. JCV thanks the Instituto de Física Teórica UAM-CSIC for providing a splendid working environment at Madrid, during the late stages of writing.

The project has received funding from the European Union's Horizon 2020 research and innovation programme under the Marie Skłodowska-Curie grant agreement No. 690575. JMG-B, FL and PV acknowledge the support of the COST action QSPACE; FL and PV acknowledge as well the Iniziativa Specifica GeoSymQFT of the INFN. FL acknowledges the Spanish MINECO under project MDM-2014-0369 of ICCUB (Unidad de Excelencia 'María de Maeztu'). JMG-B received funding from Project FPA2015-65745-P of MINECO/Feder. JCV acknowledges support from the Vicerrectoría de Investigación of the Universidad de Costa Rica.

\section{References}

[1] E. P. Wigner, “On unitary representations of the inhomogeneous Lorentz group”, Ann. Math. 40 (1939), 149-204.

[2] A. A. Kirillov, Lectures on the Orbit Method, American Mathematical Society, Providence, RI, 2004.

[3] J. F. Cariñena, J. M. Gracia-Bondía and J. C. Várilly, "Relativistic quantum kinematics in the Moyal representation", J. Phys. A 23 (1990), 901-933.

[4] Z. Li, "Coadjoint orbits and induced representations", Ph. D. thesis, MIT, 1993.

[5] Ph. Schuster and N. Toro, "On the theory of continuous spin particles: wavefunctions and soft-factor scattering amplitudes", JHEP 1309 (2013), 104.

[6] Ph. Schuster and N. Toro, "On the theory of continuous-spin particles: helicity correspondence in radiation and forces”, JHEP 1309 (2013), 105.

[7] Ph. Schuster and N. Toro, "A gauge field theory of continuous spin particles", JHEP 1310 (2013), o61.

[8] Ph. Schuster and N. Toro, "Continuous-spin particle field theory with helicity correspondence”, Phys. Rev. D 91 (2015), 025023 .

[9] T. Rempel and L. Freidel, “A bilocal model for the relativistic spinning particle”, Phys. Rev. D 95 (2017), 104014.

[10] X. Bekaert and E. Skvortsov, "Elementary particles with continuous spin”, Int. J. Mod. Phys. A 32 (2017), 1730019 .

[11] M. Najafizadeh, "Modified Wigner equations and continuous spin gauge field", Phys. Rev. D 97 (2018), o65009.

[12] K.-H. Rehren, "Pauli-Lubański limit and stress-energy tensor for infinite-spin fields", JHEP 1711 (2017), 130.

[13] M. Duflo and M. Vergne, "Une propriété de la representation coadjointe d'une algèbre de Lie", C. R. Acad. Sci. Paris 268A (1969), 583-585.

[14] A. S. Wightman, “On the localizability of quantum mechanical systems”, Rev. Mod. Phys. 34 (1962), 845-872.

[15] J. Schwinger, Particles, Sources and Fields, Addison-Wesley, Reading, MA, 1970. 
[16] A. P. Balachandran, G. Marmo, A. Simoni, A. Stern and F. Zaccaria, "On a classical description of massless particles", Proceedings of the ISAQTP-Shanxi (1992); pp. 396-402.

[17] L. J. Boya, J. F. Cariñena and M. Santander, "On the continuity of boosts for each orbit", Commun. Math. Phys. 37 (1974), 331-334.

[18] E. P. Wigner, “Relativistische Wellengleichungen”, Z. Physik 124 (1947), 665-684.

[19] J. M. Gracia-Bondía, A. Jenkins and J. C. Várilly, in preparation.

[20] S. T. Ali, A. E. Krasowska and R. Murenzi, "Wigner functions from the two-dimensional wavelet group", J. Opt. Soc. Am. A 17 (2000), 2277-2287.

[21] E. C. G. Sudarshan and N. Mukunda, Classical Dynamics: A Modern Perspective, Wiley, New York, 1974.

[22] L. Bel and J. Martín, "Predictive relativistic mechanics of $N$ particles with spin", Ann. Inst. Henri Poincaré A 33 (1980), 409-442.

[23] V. Bargmann and E. P. Wigner, "Group theoretical discussion of relativistic wave equations", Proc. Natl. Acad. Sci. USA 34 (1948), 211-223.

[24] H. Bacry, Localizability and Space in Quantum Physics, Springer, Berlin, 1988.

[25] M. A. Soloviev, "Weyl correspondence for a charged particle in the field of a magnetic monopole", Theor. Math. Phys. 187 (2016), 782-795.

[26] M. A. Soloviev, "Dirac's monopole, quaternions, and the Zassenhaus formula”, Phys. Rev. D 94 (2016), 105021.

[27] M. A. Soloviev, "Dirac's magnetic monopole and the Kontsevich star product", J. Phys. A: Math. Theor. 51 (2018), 095205 .

[28] G. G. Emch and A. Z. Jadczyk, "Weakly projective representations, quaternions and monopoles", in Stochastic Processes, Physics and Geometry: New Interplays, II, F. Gesztesy et al, eds., CMS Conference Proceedings 29, AMS, Providence, RI, 2000; pp. 157-164.

[29] J. F. Cariñena, J. M. Gracia-Bondía, F. Lizzi, G. Marmo and P. Vitale, "Monopole-based quantization: a programme", in: Mathematical Physics and Field Theory: Julio Abad, in memoriam, M. Asorey, J. V. GarcíaEsteve, M. Fernández-Rañada and J. Sesma, eds., Prensas Universitarias de Zaragoza, 2009; pp. 167-176.

[30] J. F. Cariñena, J. M. Gracia-Bondía, F. Lizzi, G. Marmo and P. Vitale, "Star-product in the presence of a monopole", Phys. Lett. A 374 (2010), 3614-3618.

[31] M. Măntoiu and R. Purice, “The magnetic Weyl calculus”, J. Math. Phys. 45 (2004), 1394-1417.

[32] M. V. Karasev and T. A. Osborn, "Cotangent bundle quantization: entangling of metric and magnetic field", J. Phys. A: Math. Gen. 38 (2005), 8549-8578.

[33] R. Abraham and J. E. Marsden, Foundations of Mechanics, Addison-Wesley, Redwood City, CA, 1987.

[34] O. L. Kurnyavko and I. V. Shirokov, "Construction of invariants of the coadjoint representation of Lie groups using linear algebra methods", Theor. Math. Phys. 188 (2016), 965-979.

[35] M. Flato, D. Sternheimer and C. Frønsdal, "Difficulties with massless particles?" Commun. Math. Phys. 9o (1983), 563-573.

[36] X. Bekaert, M. Najafizadeh and M. R. Setare, "A gauge field theory of fermionic continuous-spin particles", Phys. Lett. B 760 (2016), 320-323.

[37] M. V. Khabarov and Yu. M. Zinoviev, "Infinite (continuous) spin fields in the frame-like formalism", Nucl. Phys. B 928 (2018), 182-216. 